\title{
Spectroscopic and Excited-State Properties of Luminescent Rhenium(I ) N-Heterocyclic Carbene Complexes Containing Aromatic Diimine Ligands
}

\author{
Wen-Mei Xue, Michael Chi-Wang Chan, Zhong-Min Su, Kung-Kai Cheung, \\ Shiuh-Tzung Liu, ${ }^{\dagger}$ and Chi-Ming Che* \\ Department of Chemistry, The University of Hong Kong, Pokfulam Road, Hong Kong
}

Received October 16, 1997

\begin{abstract}
Rhenium(I) N-heterocydic carbene complexes of the type $\left.\left[\mathrm{HNCH} \mathrm{CH}_{2} \mathrm{NHCRe} \mathrm{L}-\mathrm{L}\right)(\mathrm{CO})_{3}\right]^{+}$ have been prepared, where $L-L=4,4^{\prime}$-dimethoxy-2,2'-bipyridine (2), 4,4'-bis(tert-butyl)2,2'-bipyridine (3), 2,2'-bipyridine (4), 4,4'-dichloro-2,2'-bipyridine (5), 4,4'-bis(carbomethoxy)2,2'-bipyridine (6), 5-phenyl-1,10-phenanthrol ine (7), and o-phenylenebis(di phenyl phosphine) (8). The molecular structures of 4, 6, and $\mathbf{8}$ have been determined by X-ray analyses and show Re-C(carbene) bond distances of 2.171(7), 2.163(4), and 2.199(6) $\AA$, respectively. HF$\mathrm{SCF}$ and $\mathrm{MP}_{2}$ calculations on the model compound $\left[\mathrm{HNCH} \mathrm{CH}_{2} \mathrm{NHCRe}(\mathrm{NHCHCHNH})-\right.$ $\left.(\mathrm{CO})_{3}\right]^{+}(\mathbf{4 m})$ show that the HOMO is nonbonding $\mathrm{d}(\mathrm{Re})$ and the LUMO is mainly $\pi^{*}$ (diimine) with partial $\mathrm{p}_{\mathrm{z}}$ (carbene) character. CIS calculations on the excited state of optimized $\mathbf{4 m}$ suggest that the lowest energy absorption originates from a HOMO to LUMO spin-forbidden transition. Complexes 2-7 are emissive at room temperature and $77 \mathrm{~K}$. The roomtemperature and $77 \mathrm{~K}$ luminescence data of 2-6 are consistent with emission from a ${ }^{3}$ MLCT state. The nature of the emission of 7 at room temperature is also ${ }^{3} \mathrm{MLCT}$ but changes to IL at $77 \mathrm{~K}$. Complex 8 does not emit at room temperature, but at $77 \mathrm{~K}$, the IL $\pi(\mathrm{pdpp})$ $\rightarrow \pi^{*}$ (pdpp) emission is observed. The combination of detailed spectroscopic studies and theoretical calculations reveal that the emitting state at room temperature is ${ }^{3}\left[d(\operatorname{Re}) \rightarrow \pi^{*}\right.$ (diimine)], with the latter exhibiting partial $\sigma^{*}$ (carbene) parentage. The excited-state energies and redox potentials can be tuned using diimine ligands with varying electrondonating/accepting abilities.
\end{abstract}

\section{Introduction}

Studies on transition-metal carbene complexes have given prominence to their stoichiometric and catalytic reactivities and to their important roles in a number of organic transformations. ${ }^{1}$ In contrast, little attention has been devoted to their photophysical and -chemical properties, ${ }^{2}$ even though photolysis might be expected to alter the reactivity of the carbene ligand through population of charge-transfer excited states. The photochemistry of pentacarbonyltungsten and -chromium carbene complexes have been extensively studied. ${ }^{3}$ Geoffroy and co-workers reported that the photolysis of $\mathrm{W}(\mathrm{CO})_{5}\{\mathrm{C}(\mathrm{OMe}) \mathrm{Ph}\}$ resulted in $\mathrm{CO}$ elimination as the only detectable photoreaction. ${ }^{2 a}$ This reaction was proposed to proceed via ligand-field excited states with

\footnotetext{
† Department of Chemistry, National Taiwan University, Taipei, Taiwan.

(1) Dötz, K. H.; Fischer, H.; Hofmann, P.; Kreissl, F. R.; Schubert, U.; Weiss, K. Transition Metal Carbene Complexes; Verlag Chemie: Weinheim, Germany, 1983.

(2) (a) Foley, H. C.; Strubinger, L. M.; Targos, T. S.; Geoffroy, G. L. J . Am. Chem. Soc. 1983, 105, 3064. (b) Bell, S. E. J .; Gordon, K. C.; McGarvey, J . J . J. Am. Chem. Soc. 1988, 110, 3107. (c) Rooney, A. D.; McGarvey, J .J .; Gordon, K. C.; McNicholl, R. A.; Schubert, U.; Hepp, W. Organometallics 1993, 12, 1277. (d) Rooney, A. D.; McGarvey, J . J .; Gordon, K. C. Organometallics 1995, 14, 107. (e) For recent review, see: Hegedus, L. S. ComprehensiveOrganometallic Chemistry II.; Abel, E. W., Stone, F. G. A., Wilkinson, G., Eds., Pergamon: Oxford, 1995; Vol. 12, p 549.
}

the lowest lying $\mathrm{W} \rightarrow \pi^{*}$ (carbene) charge-transfer state being inactive with respect to CO loss. A large body of work describing the photochemistry of chromium carbene complexes has been reported by Hegedus and coworkers. ${ }^{4}$

To gain insight into the photochemical reactions of carbene complexes, it is necessary to characterize the lowest-lying electronic excited states and their photophysical deactivation mechanisms. Emission spectroscopy often provides a highly sensitive and effective way to identify and study the lowest-energy excited states, ${ }^{5}$ but the first example of a Iuminescent carbene complex has only appeared recently. ${ }^{6}$

(3) (a) Fischer, E. O.; Fischer, H. Chem. Ber. 1974, 107, 657. (b) Casey, C. P.; Shusterman, A. J . J . Mol. Catal. 1970, 8, 1. (c) Casey, C. P.; Shusterman, A. J .; Vollendorf, N. W.; Haller, K. J . J . Am. Chem. Soc. 1982, 104, 2417. (d) Dahlgren, R. M.; Zink, J I I Inorg. Chem. 1977, 16, 3154. (e) Edwards, B. H.; Rausch, M. D. J . Organomet. Chem. 1981, 210, 91.

(4) (a) McGuire, M. A.; Hegedus, L. S. J . Am. Chem. Soc. 1982, 104, 5538. (b) Hegedus, L. S.; McGuire, M. A.; Schultze, L. M.; Yujin, C.; Anderson, O. P. J . Am. Chem. Soc. 1984, 106, 2680. (c) Borel, C.; Hegedus, L. S.; Krebs, J .; Satoh, Y.J. . Am. Chem. Soc. 1987, 109, 1101. (d) Hegedus, L. S.; deWeck, G.; D'Andrea, S. J . Am. Chem. Soc. 1988 110, 2122. (e) Lastra, E.; Hegedus, L. S. J . Am. Chem. Soc. 1993, 115, 87. (f) Hegedus, L. S. Acc. Chem. Res. 1995, 28, 299. (g) Hegedus, L. S. Tetrahedron 1997, 53, 4105.

(5) Lees, A. J. Chem. Rev. 1987, 87, 711.

(6) Lai, S. W.; Chan, M. C. W.; Cheung, K. K.; Che, C. M. Angew. Chem., Int. Ed. Engl. 1998, 37, 182. 
Scheme 1

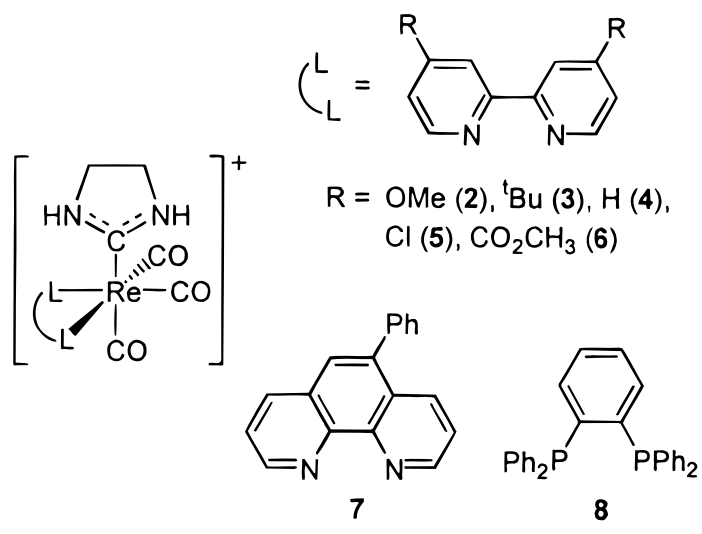

We now report on the luminescence of rhenium(I) $\mathrm{N}$-heterocyclic carbene derivatives with diimine or phosphine ligands, as depicted in Scheme 1. Some of the molecular structures are determined by X-ray crystal analyses. Assignment of the lowest-lying excited state is based on photophysical studies and molecular orbital calculations, and subtle tuning of the excitedstate energies and redox potentials is also demonstrated.

\section{Experimental Section}

General Procedures. $\mathrm{Re}(\mathrm{CO})_{5} \mathrm{Cl}$ (Strem), 2,2'-bipyridine (bpy, Aldrich), 5-phenyl-1,10-phenanthrol ine (Phphen, GFS), and o-phenylenebis(diphenyl phosphine) (pdpp, Strem) were used as received. 4,4'-Dimethoxy-2,2'-bipyridine $\left((\mathrm{MeO})_{2-}\right.$ bpy), ${ }^{7} 4,4^{\prime}$-bis(tert-butyl)-2,2'-bipyridine ('B ${ }^{2}$-bpy), ${ }^{8} 4,4^{\prime}$-dichloro2,2'-bipyridine $\left(\mathrm{Cl}_{2}\right.$-bpy), ${ }^{9}$ 4,4'-bis(carbomethoxy)-2,2'-bipyri-

dine $\left(\left(\mathrm{MeO}_{2} \mathrm{C}\right)_{2}\right.$-bpy), ${ }^{9}$ and $\mathrm{Br}(\mathrm{CO})_{4} \mathrm{ReCNHCH}_{2} \mathrm{CH}_{2} \mathrm{NH}$ (1) ${ }^{10}$ were prepared by literature methods. The dichloromethane for the photophysical studies was washed with concentrated sulfuric acid, 10\% sodium hydrogen carbonate, and water, dried by cal cium chloride, and distilled over cal cium hydride. The acetonitrile for the photophysics and electrochemistry was distilled over potassium permanganate and calcium hydride. The other solvents used were of analytical grade.

Infrared spectra were recorded with $\mathrm{KBr}$ disks on a BIORAD FTS165 FT-IR spectrophotometer. Fast atom bombardment (FAB) mass spectra were obtained on a Finnigan Mat 95 mass spectrometer. Elemental analyses were performed by Butterworth Laboratory, U.K. ${ }^{1} \mathrm{H},{ }^{13} \mathrm{C}$, and ${ }^{31} \mathrm{P} N M R$ measurements were performed on a J EOL 270, Bruker DRX 300 , or $500 \mathrm{MHz}$ FT-NMR spectrometer with TMS $\left({ }^{1} \mathrm{H}\right.$ and ${ }^{13} \mathrm{C}$ ) and $\mathrm{H}_{3} \mathrm{PO}_{4}\left({ }^{31} \mathrm{P}\right)$ as the internal reference. UV-vis absorption spectra were obtained on a Milton Roy Spectronic 3000 diode-array spectrophotometer.

Cyclic voltammetry was performed with a Princeton Applied Research (PAR) model 175 universal programmer and a model 273 potentiostat. A standard two-compartment cell was used with glassy carbon as the working el ectrode, $\mathrm{Ag}-\mathrm{AgNO}_{3}$ (0.1 mol $\mathrm{dm}^{-3}$ in acetonitrile) as the reference electrode, and platinum wire as the counter electrode. The supporting electrolyte was $\mathrm{n}$-tetrabutylammonium hexafluorophosphate $\left(0.1 \mathrm{~mol} \mathrm{dm}^{-3}\right) . \quad \mathrm{Cp}_{2} \mathrm{Fe}^{+/ 0}$ was added as an internal standard.

(7) Maerker, G.; Case, F. H. J . Am. Chem. Soc. 1958, 80, 2745.

(8) Chen, T. Y. Ph.D. Thesis, National Taiwan University, 1993

(9) Cook, M. J .; Lewis, A. P.; McAnliffe, G. S. G.; Skarda, V.; Thomson, A. J .; Glasper, J . L.; Robbins, D. J . J . Chem. Soc., Perkin Trans. II 1984, 1293.

(10) Liu, C. Y.; Chen, D. Y.; Lee, G. H.; Peng, S. M.; Liu, S. T. Organometallics 1996, 15, 1055.
Emission and Lifetime Measurements. Steady-state emission spectra were recorded on a SPEX 1681 FLOUROLOG-2 series F111Al spectrophotometer. Low- temperature (77 K) emission spectra for glasses and solid-state samples were recorded in 5-mm diameter quartz tubes which were placed in a liquid nitrogen Dewar equipped with quartz windows. The emission spectra were corrected for monochromator and photomultiplier efficiency and for xenon lamp stability.

The absol ute emission quantum yield was measured by the method of Demas and Crosby ${ }^{11}$ using quinine sulfate in $0.1 \mathrm{~N}$ sulfuric acid as the standard. Sample and standard solutions were degassed with at least three freeze-pump-thaw cycles. The quantum yield of the sample was determined by

$$
\Phi_{\mathrm{s}}=\Phi_{\mathrm{r}}\left(\mathrm{B}_{\mathrm{r}} / \mathrm{B}_{\mathrm{s}}\right)\left(\mathrm{n}_{\mathrm{s}} / \mathrm{n}_{\mathrm{r}}\right)^{2}\left(\mathrm{D}_{\mathrm{s}} / \mathrm{D}_{\mathrm{r}}\right)
$$

where the subscripts $s$ and $r$ refer to sample and reference standard solution, respectively; $\mathrm{n}$ is the refractive index of the solvents; $D$ is the integrated intensity, and $\Phi$ is the luminescence quantum yield. The quantity $B$ is calculated by

$$
\mathrm{B}=1-10^{-\mathrm{AL}}
$$

where $A$ is the absorbance at the excitation wavel ength and $L$ is the optical path length.

Emission lifetimes and flash-photolysis measurements were performed with a Quanta Ray DCR-3 pulsed Nd:YAG laser system (pulse output $355 \mathrm{~nm}, 8 \mathrm{~ns}$ ). The emission signals were detected by a Hamamatsu R928 photomultiplier tube and recorded on a Tektronix model 2430 digital oscilloscope.

Syntheses. [ $\left.\mathrm{HNCH}_{2} \mathrm{CH}_{2} \mathrm{NHCRe}(\mathrm{L}-\mathrm{L})(\mathrm{CO})_{3}\right]^{+}(\mathrm{L}-\mathrm{L}=$ 4,4-X $\mathbf{X}_{2}$-bpy, $\mathrm{X}=\mathrm{OMe}$ (2), tBu (3), $\mathrm{H}$ (4), $\mathrm{Cl}$ (5), $\mathrm{CO}_{2} \mathrm{Me}$ (6)). Complex $1(0.10 \mathrm{~g}, 0.23 \mathrm{mmol})$ and $\mathrm{L}-\mathrm{L}(0.27 \mathrm{mmol})$ in benzene $\left(10 \mathrm{~cm}^{3}\right)$ were refluxed for $4 \mathrm{~h}$ to give a yellow or orange precipitate. Upon cooling, the resultant solid was collected and washed with benzene and diethyl ether. Recrystallization by diffusion of di ethyl ether into an acetonitrile solution afforded crystals of the complexes $\left[\mathrm{HNCH}_{2} \mathrm{CH}_{2}\right.$ $\left.\mathrm{NHCRe}(\mathrm{L}-\mathrm{L})(\mathrm{CO})_{3}\right] \mathrm{Br}$. The perchlorate salt was prepared by metathesis of the bromide salt in methanol using lithium perchlorate, and crystals were obtained by diffusion of diethyl ether into an acetonitrile solution.

2: Yellow crystals of the $\mathrm{ClO}_{4}^{-}$salt, yield $0.12 \mathrm{~g}, 79 \%$. IR $\left(v_{\mathrm{CO}}, \mathrm{Cm}^{-1}\right): 2022,1924,1895$. MS (positive FAB): $\mathrm{m} / \mathrm{z} 557$ $\left(\mathrm{M}^{+}\right), 529\left(\mathrm{M}^{+}-\mathrm{CO}\right)$. Anal. Calcd for $\mathrm{C}_{18} \mathrm{H}_{18} \mathrm{~N}_{4} \mathrm{O}{ }_{9} \mathrm{ClRe}: \mathrm{C}$, 32.96; $\mathrm{H}, 2.77 ; \mathrm{N}, 8.54$. Found: $\mathrm{C}, 32.66 ; \mathrm{H}, 2.78 ; \mathrm{N}, 8.20 .{ }^{1} \mathrm{H}$ NMR $\left(\mathrm{CD}_{3} \mathrm{CN}\right): \delta 3.30\left(\mathrm{~s}, 4, \mathrm{CH}_{2}\right), 4.08\left(\mathrm{~s}, 6, \mathrm{CH}_{3} \mathrm{O}\right), 6.80(\mathrm{~s}$, $\mathrm{br}, 2, \mathrm{NH}), 7.20(\mathrm{dd}, 2$, J нн $=6.5,2.7 \mathrm{~Hz}, \mathrm{py} \mathrm{H}), 8.03(\mathrm{~d}, 2$, J нн $=2.7 \mathrm{~Hz}$, py $\mathrm{H}), 8.83(\mathrm{~d}, 2, \mathrm{~J}$ нн $=6.5 \mathrm{~Hz}$, py $\mathrm{H}) .{ }^{13} \mathrm{C} \mathrm{NMR}$ $\left(\mathrm{CD}_{3} \mathrm{CN}\right): \delta 45.2\left(\mathrm{CH}_{2}\right), 58.1\left(\mathrm{CH}_{3} \mathrm{O}\right), 112.2,115.0,155.8,158.3$, 169.3 (py C), 194.2 (carbene C), 197.6 (ax-CO), 201.6 (eq-CO).

3: Yellow crystals of the $\mathrm{ClO}_{4}^{-}$salt, yield $0.14 \mathrm{~g}, 86 \%$. IR $\left(v_{\mathrm{CO}}, \mathrm{cm}^{-1}\right): 2033,1924,1903$. MS (positive FAB): $\mathrm{m} / \mathrm{z} 609$ $\left(\mathrm{M}^{+}\right), 581\left(\mathrm{M}^{+}-\mathrm{CO}\right)$. Anal. Calcd for $\mathrm{C}_{24} \mathrm{H}_{30} \mathrm{~N}_{4} \mathrm{O}_{7} \mathrm{ClRe}: \mathrm{C}$, 40.70; $\mathrm{H}, 4.27 ; \mathrm{N}, 7.91$. Found: $\mathrm{C}, 40.92 ; \mathrm{H}, 4.52 ; \mathrm{N}, 8.15 .{ }^{1} \mathrm{H}$ NMR $\left(\mathrm{CD}_{3} \mathrm{CN}\right): \delta 1.46\left(\mathrm{~s}, 18, \mathrm{CH}_{3}\right), 3.30\left(\mathrm{~s}, 4, \mathrm{CH}_{2}\right), 6.63(\mathrm{~s}$, $\mathrm{br}, 2, \mathrm{NH}), 7.67(\mathrm{dd}, 2$, J нн $=1.9,5.9 \mathrm{~Hz}, \mathrm{py} \mathrm{H}), 8.43(\mathrm{~d}, 2$, J нн $=1.9 \mathrm{~Hz}$, py H), $8.91(\mathrm{~d}, 2, \mathrm{~J} \mathrm{HH}=5.9 \mathrm{~Hz}$, py H$) .{ }^{13} \mathrm{C} \mathrm{NMR}$ $\left(\mathrm{CD}_{3} \mathrm{CN}\right): \delta 30.5\left(\mathrm{CH}_{3}\right), 36.7\left(\mathrm{CMe}_{3}\right), 45.3\left(\mathrm{CH}_{2}\right), 122.8,126.2$ 154.3, 156.6, 165.9 (py C), 193.7 (carbene C), 197.6 (ax-CO), 201.1 (eq-CO).

4: Yellow crystals of the $\mathrm{Br}^{-}$salt, yield $0.11 \mathrm{~g}, 83 \%$. IR ( $v_{\mathrm{CO}}$, $\mathrm{cm}^{-1}$ ): 2022, 1936, 1905. MS (positive FAB): $\mathrm{m} / \mathrm{z} 497\left(\mathrm{M}^{+}\right.$), $469\left(\mathrm{M}^{+}-\mathrm{CO}\right)$. Anal. Calcd for $\mathrm{C}_{16} \mathrm{H}_{14} \mathrm{~N}_{4} \mathrm{O}_{3}$ BrRe: C, 33.34; $\mathrm{H}, 2.45 ; \mathrm{N}, 9.72$. Found: $\mathrm{C}, 33.12 ; \mathrm{H}, 2.53 ; \mathrm{N}, 10.01$. ${ }^{1} \mathrm{H}$ NMR $\left(\mathrm{CD}_{3} \mathrm{CN}\right): \delta 3.28\left(\mathrm{~s}, 4, \mathrm{CH}_{2}\right), 6.80(\mathrm{~s}, \mathrm{br}, 2, \mathrm{NH}), 7.68(\mathrm{~m}, 2, \mathrm{py}$

(11) Demas, J . N.; Crosby, G. A. J . Phys. Chem. 1971, 75, 991. 
H), $8.23\left(\mathrm{td}, 2, \mathrm{~J}_{\mathrm{HH}, \mathrm{t}}=8.0 \mathrm{~Hz}, \mathrm{~J}_{\mathrm{H}, \mathrm{d}}=1.5 \mathrm{~Hz}, \mathrm{py} \mathrm{H}\right), 8.51$ (d, 2 , J Hн $=8.0 \mathrm{~Hz}$, py H), $9.09\left(\mathrm{~m}, 2\right.$, py H). ${ }^{13} \mathrm{C} \mathrm{NMR}\left(\mathrm{CD}_{3} \mathrm{CN}\right)$ : $\delta 45.2\left(\mathrm{CH}_{2}\right), 125.5,129.2,140.9,154.9,156.6$ (py C), 193.5 (carbene C), 197.4 (ax-CO), 200.8 (eq-CO).

5: Yellow crystals of the $\mathrm{ClO}_{4}{ }^{-}$salt, yield $0.12 \mathrm{~g}, 78 \%$. IR $\left(v_{\mathrm{CO}}, \mathrm{cm}^{-1}\right)$ : 2028, 1941, 1891. MS (positive FAB): m/z 565 $\left(\mathrm{M}^{+}\right), 537\left(\mathrm{M}^{+}-\mathrm{CO}\right)$. Anal. Calcd for $\mathrm{C}_{16} \mathrm{H}_{12} \mathrm{~N}_{4} \mathrm{O}_{7} \mathrm{Cl}_{3} \mathrm{Re}: \mathrm{C}$, 28.90; $\mathrm{H}, 1.82 ; \mathrm{N}, 8.43$. Found: $\mathrm{C}, 28.52 ; \mathrm{H}, 1.74 ; \mathrm{N}, 8.81 .{ }^{1} \mathrm{H}$ NMR $\left(\mathrm{CD}_{3} \mathrm{CN}\right): \delta 3.29\left(\mathrm{~s}, 4, \mathrm{CH}_{2}\right), 6.71(\mathrm{~s}, \mathrm{br}, 2, \mathrm{NH}), 7.74$ (dd, 2, J нн $=6.0,2.2 \mathrm{~Hz}$, py H), 8.60 (s, 2, py H), 8.98 (d, 2, $\mathrm{J} \mathrm{HH}=6.0 \mathrm{~Hz}$, py H$) .{ }^{13} \mathrm{C} \mathrm{NMR}\left(\mathrm{CD}_{3} \mathrm{CN}\right): \delta 45.2\left(\mathrm{CH}_{2}\right), 126.5$, 129.6, 148.7, 155.7, 157.2 (py C), 192.9 (carbene C), 197.0 (axCO), 200.2 (eq-CO).

6: Orange crystals of the $\mathrm{ClO}_{4}^{-}$salt, yield $0.13 \mathrm{~g}, 79 \%$. IR $\left(v_{\mathrm{CO}}, \mathrm{cm}^{-1}\right): 2031,1941,1909$. MS (positive FAB): $\mathrm{m} / \mathrm{z} 613$ $\left(\mathrm{M}^{+}\right), 585\left(\mathrm{M}^{+}-\mathrm{CO}\right)$. Anal. Calcd for $\mathrm{C}_{20} \mathrm{H}_{18} \mathrm{~N}_{4} \mathrm{O}_{11} \mathrm{ClRe}$ : C, 33.71; $\mathrm{H}, 2.55 ; \mathrm{N}, 7.87$. Found: $\mathrm{C}, 33.71 ; \mathrm{H}, 2.38 ; \mathrm{N}, 7.73 .{ }^{1} \mathrm{H}$ NMR $\left(\mathrm{CD}_{3} \mathrm{CN}\right): \delta 3.26\left(\mathrm{~s}, 4, \mathrm{CH}_{2}\right), 4.04\left(\mathrm{~s}, 6, \mathrm{CH}_{3}\right), 6.53(\mathrm{~s}, \mathrm{br}$, 2, NH), 8.11 (dd, 2, J нн $=5.6,1.4 \mathrm{~Hz}$, py H), $8.97(\mathrm{~d}, 2, \mathrm{~J} \mathrm{нн}=$ $1.4 \mathrm{~Hz}$, py H), $9.21(\mathrm{~d}, 2$, J нH $=5.6 \mathrm{~Hz}, \mathrm{py} \mathrm{H}) .{ }^{13} \mathrm{C} \mathrm{NMR}\left(\mathrm{CD}_{3^{-}}\right.$ $\mathrm{CN}): \delta 45.3\left(\mathrm{CH}_{2}\right), 54.2\left(\mathrm{CH}_{3}\right), 125.0,128.3,141.8,155.8,157.3$ (py C), $164.8\left(\mathrm{CO}_{2}\right), 192.5$ (carbene C), 197.0 (ax-CO), 199.9 (eq-CO).

[HNCH $\left.\mathrm{CH}_{2} \mathrm{NHCRe}(\mathrm{Phphen})(\mathrm{CO})_{3}\right] \mathrm{ClO}_{4}$ (7). A mixture of $1(0.10 \mathrm{~g}, 0.23 \mathrm{mmol})$ and Phphen $(0.07 \mathrm{~g}, 0.27 \mathrm{mmol})$ in benzene $(10 \mathrm{~mL})$ was heated to reflux for $6 \mathrm{~h}$. The resultant orange solution was evaporated to dryness, and the residue was dissolved in $\mathrm{CH}_{2} \mathrm{Cl}_{2}(5 \mathrm{~mL})$. Diethyl ether was added to precipitate the bromide salt. The yellow crystalline perchlorate salt was obtained as for complexes 2-6: yield $0.13 \mathrm{~g}, 81 \%$. IR $\left(v_{\mathrm{CO}}, \mathrm{cm}^{-1}\right)$ : 2023, 1909 (br). MS (positive FAB): m/z 597 $\left(\mathrm{M}^{+}\right), 569\left(\mathrm{M}^{+}-\mathrm{CO}\right)$. Anal. Calcd for $\mathrm{C}_{24} \mathrm{H}_{18} \mathrm{~N}_{4} \mathrm{O}_{7} \mathrm{CIRe}: \mathrm{C}$, 41.41; $\mathrm{H}, 2.61 ; \mathrm{N}, 8.05$. Found: $\mathrm{C}, 41.32 ; \mathrm{H}, 2.36 ; \mathrm{N}, 7.92 .{ }^{1} \mathrm{H}$ NMR $\left(\mathrm{CD}_{3} \mathrm{CN}\right): \delta 3.19\left(\mathrm{~s}, 4, \mathrm{CH}_{2}\right), 6.98(\mathrm{~s}, \mathrm{br}, 2, \mathrm{NH}), 7.62(\mathrm{~m}$, 5, aryl H), $7.99(\mathrm{dq}, 2, \mathrm{~J} \mathrm{Hн,d}=13.9 \mathrm{~Hz}, \mathrm{~J} \mathrm{Hн,q}=5.2 \mathrm{~Hz}$, aryl $\mathrm{H}), 8.16(\mathrm{~s}, 1$, aryl H), $8.64(\mathrm{dd}, 1, \mathrm{~J} \mathrm{Hн}=8.6,1.3 \mathrm{~Hz}$, aryl H), 8.80 (dd, 1, J нн $=7.7,1.3 \mathrm{~Hz}$, aryl H), 9.53 (qd, 2, J нн,q $=5.2$ $\mathrm{Hz}$, J $\mathrm{HH}, \mathrm{d}=1.3 \mathrm{~Hz}$, aryl H). ${ }^{13} \mathrm{C} N M R\left(\mathrm{CD}_{3} \mathrm{CN}\right): \delta 45.1\left(\mathrm{CH}_{2}\right)$, $127.5,128.0,128.5,129.9,130.1,131.0,131.5,137.9,138.5$, 139.9, 141.5, 146.9, 147.9, 155.5 (aryl C), 193.5 (carbene C), 197.4 (ax-CO), 200.6 (eq-CO).

[HNCH $\left.\left.\mathbf{C H}_{2} \mathrm{NHCRe} \mathrm{Cdpp}\right)(\mathrm{CO})_{3}\right] \mathrm{Br}$ (8). A benzene solution $(10 \mathrm{~mL})$ of $\mathbf{1}(0.10 \mathrm{~g}, 0.23 \mathrm{mmol})$ and pdpp $(0.12 \mathrm{~g}, 0.27$ $\mathrm{mmol}$ ) was refluxed for $4 \mathrm{~h}$ to give a white precipitate. Upon cooling to room temperature, the solid was collected and washed with benzene and diethyl ether. Recrystallization by diffusion of diethyl ether into an acetonitrile solution afforded colorless crystals: yield $0.16 \mathrm{~g}, 78 \%$. IR $\left(v_{\mathrm{CO}}, \mathrm{cm}^{-1}\right)$ : 2031 , 1958, 1931. MS (positive FAB): $\mathrm{m} / \mathrm{z} 787\left(\mathrm{M}^{+}\right), 759\left(\mathrm{M}^{+}-\mathrm{CO}\right)$. Anal. Calcd for $\mathrm{C}_{36} \mathrm{H}_{30} \mathrm{~N}_{2} \mathrm{O}_{3} \mathrm{BrP}_{2}$ Re: $\mathrm{C}, 49.89 ; \mathrm{H}, 3.49 ; \mathrm{N}, 3.23$. Found: $\mathrm{C}, 50.22 ; \mathrm{H}, 3.41 ; \mathrm{N}, 3.47 .{ }^{1} \mathrm{H} \mathrm{NMR}\left(\mathrm{CD}_{3} \mathrm{CN}\right): \delta 3.13$ $\left(\mathrm{s}, 4, \mathrm{CH}_{2}\right), 6.33(\mathrm{~s}, 2, \mathrm{NH}), 7.16(\mathrm{~m}, 4$, aryl H), $7.38(\mathrm{~m}, 6$, aryl $H)$, $7.46(m, 4$, aryl H), $7.56(m, 6$, aryl H) , $7.83(m, 2$, aryl H), $7.90\left(\mathrm{~m}, 2\right.$, aryl H). ${ }^{13} \mathrm{C}$ NMR $\left(\mathrm{CD}_{3} \mathrm{CN}\right): \delta 45.9\left(\mathrm{CH}_{2}\right), 129.3-$ $141.2\left(\mathrm{~m}\right.$, aryl C), $189.9\left(\mathrm{t},{ }^{2} \mathrm{~J} \mathrm{cP}=9.7 \mathrm{~Hz}\right.$, carbene C), $191.5(\mathrm{t}$, ${ }^{2} \mathrm{~J} \mathrm{CP}_{\mathrm{CP}} 6.6 \mathrm{~Hz}$, ax-CO), $192.2\left(\mathrm{~d},{ }^{2} \mathrm{~J} \mathrm{CP}=8.7 \mathrm{~Hz}\right.$, eq-CO), 192.5 $\left(\mathrm{d},{ }^{2} \mathrm{JP}_{\mathrm{CP}}=8.6 \mathrm{~Hz}\right.$, eq-CO). ${ }^{31} \mathrm{P} \mathrm{NMR}\left(\mathrm{CD}_{3} \mathrm{CN}\right): \delta 37.6$.

\section{X-ray Crystallography. Crystals of $\left[\mathrm{HNCH}_{2} \mathrm{CH}_{2} \mathrm{NHCRe}\right.$} (bpy) $\left.(\mathrm{CO})_{3}\right] \mathrm{Br} \cdot \mathrm{CH}_{3} \mathrm{CN} \cdot 0.5 \mathrm{H}_{2} \mathrm{O}$ (4), [ $\mathrm{HNCH}_{2} \mathrm{CH}_{2} \mathrm{NHCRe}\left(4,4^{\prime}-\right.$ $\left(\mathrm{CO}_{2} \mathrm{Me}\right)_{2}$-bpy) $\left.(\mathrm{CO})_{3}\right] \mathrm{ClO}_{4} \cdot \mathrm{H}_{2} \mathrm{O}(6)$, and $\left[\mathrm{HNCH}_{2} \mathrm{CH}_{2} \mathrm{NHCRe}\right.$ (pdpp)(CO) $\left.)_{3}\right] \mathrm{Br}$ (8) were mounted on a Rigaku AFC7R diffractometer at $301 \mathrm{~K}$ with graphite-monochromated MoK $\alpha$ radiation $(\lambda=0.71073 \AA$ ) using $\omega-2 \theta$ scans at a scan speed of $16.0^{\circ} / \mathrm{min}$ and $2 \theta_{\max }=50^{\circ}$. Intensity data were corrected for decay and Lorentz and polarization effects, and empirical absorption corrections were made based on the $\psi$-scan of six strong reflections. The structures were solved by direct methods (SIR92 ${ }^{13}$ for $\mathbf{4}$ ) or by Patterson methods (for $\mathbf{6}$ and 8), expanded by Fourier methods (PATTY ${ }^{14}$ ), and refined by full-matrix least-squares using the software package TeXsan on a Silicon Graphics Indy computer.

For complex 4, two $\mathrm{Br}$ atoms and the $\mathrm{O}$ atoms of the water mol ecule were at special positions with an occupation number of $1 / 2$. All 30 non- $\mathrm{H}$ atoms were refined anisotropically. The hydrogen atoms of the water molecule were not located. Seventeen $\mathrm{H}$ atoms were included in the calculation, and these comprised of two $\mathrm{H}$ atoms bonded to the $\mathrm{N}(3)$ and $\mathrm{N}(4)$ atoms located in the difference Fourier synthesis and $15 \mathrm{H}$ atoms at calculated positions with thermal parameters equal to 1.3 times that of the attached $\mathrm{C}$ atoms, but their positional parameters were not refined.

For complex 6, all 38 non-H atoms were refined anisotropically. The two $\mathrm{H}$ atoms bonded to the $\mathrm{N}(3)$ and $\mathrm{N}(4)$ atoms were located in the difference Fourier synthesis, and their positional parameters were refined. The hydrogen atoms of the water molecule were not located. The other $16 \mathrm{H}$ atoms at calculated positions with thermal parameters equal to 1.3 times that of the attached $\mathrm{C}$ atoms were not refined.

For complex 8, all 45 non-H atoms were refined anisotropically. The two $\mathrm{H}$ atoms bonded to the $\mathrm{N}(1)$ and $\mathrm{N}(2)$ atoms were located in the difference Fourier synthesis, and their positional parameters were refined. The hydrogen atoms of the water molecule were not located. The other $28 \mathrm{H}$ atoms at calculated positions with thermal parameters equal to 1.3 times that of the attached $C$ atoms were not refined.

The crystal data are summarized in Table 1 , and selected bond distances and angles are listed in Table 2.

Hartree-Fock Self-Consistent-Field and SecondOrder Moller-Plesset Calculations. HF-SCF ${ }^{15}$ and $\mathrm{MP}_{2}{ }^{16}$ calculations have been performed using the GAUSSIAN 94/ DFT program package ${ }^{17}$ on a Silicon Graphics Indigo 2 workstation. For the $\mathrm{Re}$ atom, the quasirelativistic (QR) pseudopotential (pp) developed by Hay and Wadt ${ }^{18}$ with 15 valence electrons (VE) and the LANL2DZ basis sets associated with the pseudopotential were adopted. The basis sets were taken as $\operatorname{Re}[8 \mathrm{~s} 6 \mathrm{p} 3 \mathrm{~d}] /(3 \mathrm{~s} 3 \mathrm{p} 2 \mathrm{~d}), \quad \mathrm{N}[10 \mathrm{~s} 5 \mathrm{p}] /(3 \mathrm{~s} 2 \mathrm{p}), \mathrm{O}[10 \mathrm{~s} 5 \mathrm{p}]$ (3s2p), C[10s5p]/(3s2p), and $H[4 s] /(2 s)$. The geometry of the calculation molecule $\left[\mathrm{HNCH}_{2} \mathrm{CH}_{2} \mathrm{NHCRe}(\mathrm{NHCHCHNH})(\mathrm{CO})_{3}\right]^{+}$(4m) was adapted from the X-ray structure of $\mathbf{4}$.

\section{Results and Discussion}

Characterization and Crystal Structures. The carbene complexes 2-7 are stable both in the solid and

(12) Freni, M.; Giusto, D.; Romiti, P. J . Inorg. Nud. Chem. 1967 29, 761.

(13) Altomare, A.; Cascarano, M.; Giacovazzo, C.; Guagliardi, A.; Burla, M. C.; Polidori, G.; Camalli, M. J . Appl. Crystallogr. 1994, 27, 435.

(14) Beurskens, P. T.; Admiraal, G.; Beurskens, G.; Bosman, W. P.; Garcia-Granda, S.; Gould, R. O.; Smits, J. M. M.; Smykalla, C. The DIRDIF program system. Technical Report of the Crystallography Laboratory; University of Nijmegen: The Netherlands, 1992.

(15) (a) Roothan, C. C. J . Rev. Mod. Phys. 1951, 23, 69. (b) Pople, J. A.; Nesbet, R. K. J . Chem. Phys. 1959, 22, 571. (c) McWeeny, R.; Dierksen, G. J . Chem. Phys. 1968, 49, 4852.

(16) (a) Head-Gorden, M.; Pople, J . A.; Frisch, M. J . Chem. Phys. Lett. 1988, 153, 503. (b) Frisch, M.J .; Head-Gorden, M.; Pople, J . A Chem. Phys. Lett. 1990, 166, 275. (c) Frisch, M. J .; Head-Gorden, M.; Pople, J. A. Chem. Phys. Lett. 1990, 166, 281.

(17) Frisch, M. J .; Trucks, G. W.; Schlegel, H. B.; Gill, P. M. W. J ohnson, B. G.; Robb, M. A.; Cheeseman, J. R.; Keith, T.; Petersson, G. A.; Montgomery, J. A.; Raghavachari, K.; Al-Laham, M. A.; Zakrzewski, V. G.; Ortiz, J. V.; Foresman, J. B.; Cioslowski, J .; Stefanov, B. B.; Nanayakkara, A.; Challacombe, M.; Peng, C. Y.; Ayala P. Y.; Chen, W.; Wong, M. W.; Andres, J . L.; Replogle, E. S.; Gomperts, R.; Martin, R. L.; Fox, D. J .; Binkley, J . S.; Defrees, D. J .; Baker, J .; Stewart, J. P.; Head-Gordon, M.; Gonzalez, C.; Pople, J . A. Gaussian 94, Revision C.3; Gaussian, Inc.: Pittsburgh, PA, 1995.

(18) (a) Hay, P. J .; Wadt, W. R. J . Chem. Phys. 1985, 82, 270. (b) Schwerdtfeger, P.; Dolg, M.; Schwarz, W. H. E.; Bowmaker, G. A.; Boyd P. D. W. J . Chem. Phys. 1989, 91, 1762. 
Table 1. Crystal Data for Complexes 4, 6, and 8

\begin{tabular}{|c|c|c|c|}
\hline & 4 & 6 & 8 \\
\hline $\begin{array}{l}\text { formula } \\
\text { fw }\end{array}$ & $\begin{array}{l}\mathrm{C}_{18} \mathrm{H}_{18} \mathrm{BrN}_{5} \mathrm{O}_{3.5} \mathrm{Re} \\
626.49\end{array}$ & $\begin{array}{l}\mathrm{C}_{20} \mathrm{H}_{20} \mathrm{ClN}_{4} \mathrm{O}_{12} \mathrm{Re} \\
730.07\end{array}$ & $\begin{array}{l}\mathrm{C}_{36} \mathrm{H}_{30} \mathrm{BrN}_{2} \mathrm{O}_{3} \mathrm{P}_{2} \mathrm{Re} \\
866.71\end{array}$ \\
\hline color & pale yellow & yellow & colorless \\
\hline cryst dimens, $\mathrm{mm}$ & $0.25 \times 0.20 \times 0.30$ & $0.20 \times 0.20 \times 0.35$ & $0.15 \times 0.05 \times 0.20$ \\
\hline cryst syst & orthorhombic & triclinic & triclinic \\
\hline space group & Ccca (No. 68) & $\mathrm{P} \overline{1}($ No. 2$)$ & $\mathrm{P} \overline{1}$ (No. 2) \\
\hline$a, \AA$ & $19.512(8)$ & $11.826(6)$ & $12.027(8)$ \\
\hline $\mathrm{b}, \AA$ & $29.865(8)$ & $12.012(6)$ & $16.75(1)$ \\
\hline$c, \AA$ & $14.523(8)$ & $11.694(5)$ & $9.582(3)$ \\
\hline$\alpha$, deg & & $117.45(3)$ & $105.26(4)$ \\
\hline$\beta$, deg & & $97.33(4)$ & 109.93(4) \\
\hline$\gamma$, deg & & $60.61(3)$ & $73.23(5)$ \\
\hline $\mathrm{V}, \AA^{3}$ & $8462(5)$ & $1274(1)$ & $1708(1)$ \\
\hline Z & 16 & 2 & 2 \\
\hline$\rho_{\text {calcd }} \mathrm{g} \mathrm{cm}^{-3}$ & 1.967 & 1.903 & 1.685 \\
\hline abs coeff, $\mathrm{cm}^{-1}$ & 76.71 & 49.45 & 48.62 \\
\hline no. of unique data collected & 4088 & 4485 & 6008 \\
\hline no. of obsd data with $\mathrm{I} \geq 3 \sigma(\mathrm{I})$ & 2584 & 4036 & 4776 \\
\hline no. of variables & 259 & 349 & 412 \\
\hline $\mathrm{R}^{\mathrm{a}}$ & 0.028 & 0.023 & 0.030 \\
\hline $\mathrm{R}_{\mathrm{w}}^{\mathrm{b}}$ & 0.039 & 0.026 & 0.034 \\
\hline goodness-of-fit, Sc & 1.89 & 1.45 & 1.25 \\
\hline$\Delta \rho\left(\max , \min , \mathrm{e} \AA^{-3}\right)$ & $+1.13,-0.67$ & $+0.62,-0.54$ & $+0.80,-1.01$ \\
\hline
\end{tabular}

Table 2. Selected Bond Distances $(\AA)$ and Angles (deg) for Complexes 4, 6, and 8

\begin{tabular}{|c|c|c|c|}
\hline \multicolumn{4}{|c|}{ Complex 4} \\
\hline $\operatorname{Re}(1)-N(1)$ & $2.178(6)$ & $\operatorname{Re}(1)-N(2)$ & $2.175(6)$ \\
\hline $\operatorname{Re}(1)$ & $1.917(9)$ & $\operatorname{Re}(1)-C(2)$ & 4(9) \\
\hline $\operatorname{Re}(1)-C(3)$ & $1.965(9)$ & $\operatorname{Re}(1)-C(4)$ & $2.171(7)$ \\
\hline$C(4)-N$ & $1.33(1)$ & t) $-N(4)$ & 1) \\
\hline $\begin{array}{l}N(1)-\operatorname{Re}(1)-\mathrm{N}(2) \\
\mathrm{N}(1)-\operatorname{Re}(1)-\mathrm{C}(2) \\
\mathrm{N}(1)-\operatorname{Re}(1)-\mathrm{C}(4) \\
\mathrm{N}(2)-\operatorname{Re}(1)-\mathrm{C}(2) \\
\mathrm{N}(2)-\operatorname{Re}(1)-\mathrm{C}(4) \\
\mathrm{C}(1)-\operatorname{Re}(1)-\mathrm{C}(3) \\
\mathrm{C}(2)-\operatorname{Re}(1)-\mathrm{C}(3) \\
\mathrm{C}(3)-\operatorname{Re}(1)-\mathrm{C}(4) \\
\operatorname{Re}(1)-\mathrm{C}(4)-\mathrm{N}(4)\end{array}$ & $\begin{array}{l}74.0(2) \\
97.6(3) \\
87.5(2) \\
171.0(3) \\
85.5(2) \\
88.4(3) \\
90.5(3) \\
178.6(3) \\
126.7(5)\end{array}$ & $\begin{array}{l}N(1)-\operatorname{Re}(1)-C(1) \\
N(1)-\operatorname{Re}(1)-C(3) \\
N(2)-\operatorname{Re}(1)-C(1) \\
N(2)-\operatorname{Re}(1)-C(3) \\
C(1)-\operatorname{Re}(1)-C(2) \\
C(1)-\operatorname{Re}(1)-C(4) \\
C(2)-\operatorname{Re}(1)-\mathrm{C}(4) \\
\operatorname{Re}(1)-\mathrm{C}(4)-\mathrm{N}(3) \\
N(3)-\mathrm{C}(4)-\mathrm{N}(4)\end{array}$ & $\begin{array}{l}173.2(3) \\
92.7(2) \\
99.2(3) \\
93.2(3) \\
89.1(4) \\
91.2(3) \\
90.9(3) \\
125.1(5) \\
108.2(6)\end{array}$ \\
\hline \multicolumn{4}{|c|}{ Complex 6} \\
\hline $\mathrm{Re}(1)-\mathrm{N}(1$ & $2.178(3)$ & $\operatorname{Re}(1)-N(2)$ & $2.172(3)$ \\
\hline $\operatorname{Re}(1)-$ & $1.908(5)$ & $(1)-C(2)$ & $8(5)$ \\
\hline $\operatorname{Re}(1)$ & $1.966(5)$ & $\mathrm{Re}(1)-C(4)$ & $2.163(4)$ \\
\hline$C(4)-N($ & $1.321(5)$ & $C(4)-N(4)$ & $1.318(5)$ \\
\hline $\begin{array}{l}\mathrm{N}(1)-\operatorname{Re}(1)-\mathrm{N}(2) \\
\mathrm{N}(1)-\operatorname{Re}(1)-\mathrm{C}(2) \\
\mathrm{N}(1)-\operatorname{Re}(1)-\mathrm{C}(4) \\
\mathrm{N}(2)-\operatorname{Re}(1)-\mathrm{C}(2) \\
\mathrm{N}(2)-\operatorname{Re}(1)-\mathrm{C}(4) \\
\mathrm{C}(1)-\operatorname{Re}(1)-\mathrm{C}(3) \\
\mathrm{C}(2)-\operatorname{Re}(1)-\mathrm{C}(3) \\
\mathrm{C}(3)-\operatorname{Re}(1)-\mathrm{C}(4) \\
\operatorname{Re}(1)-\mathrm{C}(4)-\mathrm{N}(4)\end{array}$ & $\begin{array}{l}75.0(1) \\
98.4(2) \\
83.5(1) \\
173.2(1) \\
87.5(1) \\
90.5(2) \\
90.1(2) \\
178.0(2) \\
126.2(3)\end{array}$ & $\begin{array}{l}\mathrm{N}(1)-\operatorname{Re}(1)-\mathrm{C}(1) \\
\mathrm{N}(1)-\operatorname{Re}(1)-\mathrm{C}(3) \\
\mathrm{N}(2)-\operatorname{Re}(1)-\mathrm{C}(1) \\
\mathrm{N}(2)-\operatorname{Re}(1)-\mathrm{C}(3) \\
\mathrm{C}(1)-\operatorname{Re}(1)-\mathrm{C}(2) \\
\mathrm{C}(1)-\operatorname{Re}(1)-\mathrm{C}(4) \\
\mathrm{C}(2)-\operatorname{Re}(1)-\mathrm{C}(4) \\
\mathrm{Re}(1)-\mathrm{C}(4)-\mathrm{N}(3) \\
\mathrm{N}(3)-\mathrm{C}(4)-\mathrm{N}(4)\end{array}$ & $\begin{array}{l}171.3(2) \\
94.5(2) \\
97.7(2) \\
91.9(2) \\
88.8(2) \\
91.5(2) \\
90.3(2) \\
127.1(3) \\
106.7(4)\end{array}$ \\
\hline \multicolumn{4}{|c|}{ Complex 8} \\
\hline $\operatorname{Re}(1)-$ & $2.437(1)$ & $\operatorname{Re}(1)$ & $2.444(1)$ \\
\hline $\operatorname{Re}(1)$ & $1.963(6)$ & $\operatorname{Re}(1)-C(2)$ & 1.950(6) \\
\hline $\operatorname{Re}(1)$ & $1.928(7)$ & (4) & 2.199(6) \\
\hline$C(4)-N(1)$ & $1.328(7)$ & $C(4)-N(2)$ & $1.318(7)$ \\
\hline (2) & $82.57(5)$ & )$-\operatorname{Re}(1)-C($ & $175.5(2$ \\
\hline & 7(2) & & \\
\hline$P(1)$ & $89.1(2)$ & $P(2)-\operatorname{Re}(1)-C($ & $93.3(2)$ \\
\hline $\mathrm{P}(2$ & $175.1(2)$ & $P(2)-\operatorname{Re}(1)-C(3)$ & $93.9(2)$ \\
\hline$P(2)-\operatorname{Re}(1)-C(4)$ & $89.5(2)$ & $-\operatorname{Re}(1)-C(2)$ & $91.4(2)$ \\
\hline & $87.9(2)$ & C & $89.1(2)$ \\
\hline & $87.7(2)$ & $C(2)-\operatorname{Re}(1)-C(4)$ & $89.1(2)$ \\
\hline & $175.6(2)$ & $\operatorname{Re}(1)-C(4)-N(1)$ & $129.3(4)$ \\
\hline $\operatorname{Re}(1)-C(4)-N(2)$ & $122.8(4)$ & $N(1)-C(4)-N(2)$ & $107.9(5)$ \\
\hline
\end{tabular}

solution state. Their infrared spectra show a pattern of three peaks in the carbonyl region (2022-2031,

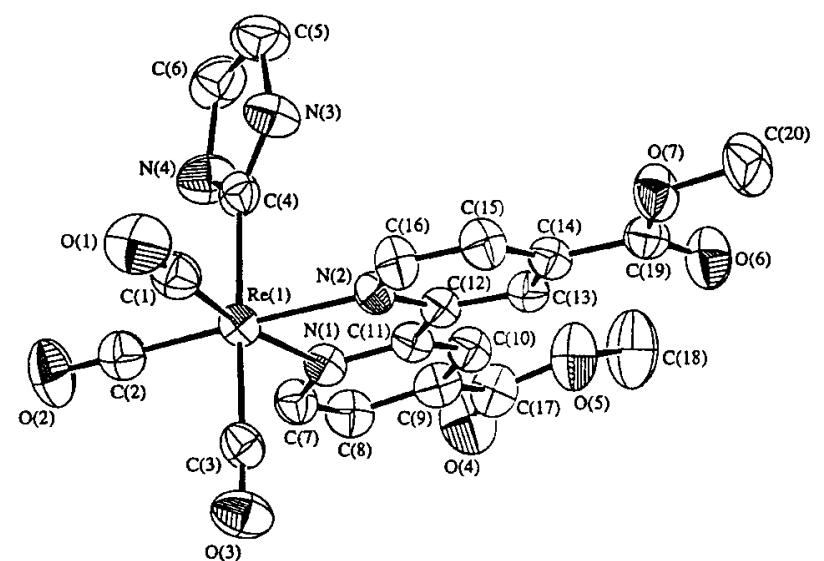

Figure 1. ORTEP plot of the cation in complex $6(40 \%$ probability ellipsoids).

1924-1941, and $1891-1909 \mathrm{~cm}^{-1}$ ) which are typical for fac-Re $(\mathrm{L}-\mathrm{L})(\mathrm{CO})_{3} \mathrm{X}$ complexes. In the ${ }^{13} \mathrm{C}$ NMR spectra, the resonances for the carbene carbon appear in the 189.9-194.2 ppm range, which is comparable to that of $192.0 \mathrm{ppm}$ in complex $\mathbf{1}$. $^{10}$

The molecular structures of $\mathbf{4}$ (see Supporting Information), 6, and 8 (Figures 1 and 2, respectively) have been determined by X-ray diffraction methods. The rhenium atom in each case has a pseudo-octahedral geometry (Table 2) with the three carbonyl ligands in a facial configuration and the carbene group trans to one of these $\left(C(3)-\operatorname{Re}(1)-C(4) 178.6(3)^{\circ}, 178.0(2)^{\circ}\right.$, and $175.6(2)^{\circ}$ for $\mathbf{4}, \mathbf{6}$, and $\mathbf{8}$, respectively). The Re$\mathrm{C}$ (carbene) $-\mathrm{N}$ angles approach $120^{\circ}$, in agreement with $\mathrm{sp}^{2}$ hybridization at the carbene carbon atoms (e.g., in complex 8, Re(1)-C(4)-N(1) 129.3(4) $)^{\circ}, \operatorname{Re}(1)-\mathrm{C}(4)-\mathrm{N}(2)$ $122.8(4)^{\circ}, \mathrm{N}(1)-\mathrm{C}(4)-\mathrm{N}(2) 107.9(5)^{\circ}$.

Previous studies revealed that the metal-carbene bond distances are perturbed by the organic substituents attached to the carbene carbon. ${ }^{1}$ In this work, the Re-C(carbene) distances (2.171(7), 2.163(4), and 2.199(6) $\AA$ for $\mathbf{4}, \mathbf{6}$, and 8, respectively) are comparable with those in other heteroatom-stabilized anal ogues such as fac- $\mathrm{ReBr}(\mathrm{CO})_{3}\left(\mathrm{CNHCH}_{2} \mathrm{CH}_{2} \mathrm{NH}\right)_{2}$ (2.14(2) and 2.17(2) 


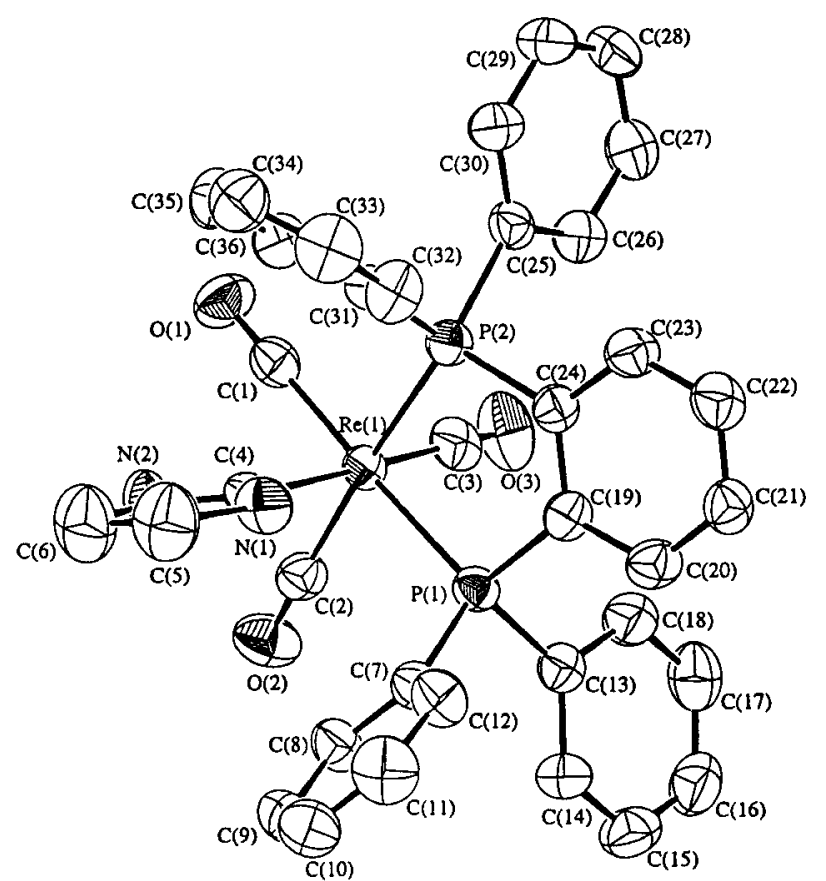

Figure 2. ORTEP plot of the cation in complex 8 (40\% probability ellipsoids).

$\AA ̊),{ }^{10} \mathrm{ReBr}(\mathrm{CO})_{4}\left(\mathrm{COCH}_{2} \mathrm{CH}_{2} \mathrm{O}\right)(2.135(13) \AA \AA),{ }^{19} \mathrm{ReBr}-$ $(\mathrm{CO})_{4}\{\mathrm{C}(\mathrm{NHPh})(\mathrm{NHCHMe})\}(2.206(10) \AA),{ }^{20}$ and $\mathrm{ReBr}-$ $(\mathrm{CO})_{3}\left(\mathrm{PPh}_{3}\right)\left\{\mathrm{C}(\mathrm{NHPh})\left(\mathrm{NHCHMe}_{2}\right)\right\}(2.215(8) \AA) .{ }^{20}$ They are longer than the $\mathrm{Re}$-alkylcarbene contact in $\mathrm{CpRe}$ $(\mathrm{NO})\left(\mathrm{PPh}_{3}\right)(\mathrm{CHPh})^{+}(1.949(6) \AA)^{21}$ but are similar to the $\mathrm{Re}-\mathrm{C}\left(\mathrm{sp}^{3}\right)$ bond in the corresponding benzyl derivative $\mathrm{CpRe}(\mathrm{NO})\left(\mathrm{PPh}_{3}\right)\left(\mathrm{CH}_{2} \mathrm{Ph}\right)^{+}(2.203(8) \AA) .{ }^{22}$ Nevertheless, the angles around the carbene atoms in 4, 6, and 8 imply that there are appreciable $\mathrm{Re}-\mathrm{C}$ (carbene) $\pi$ interactions in these complexes.

Electronic Structure and Absorption Spectra. As an aid to interpreting the electronic absorption spectra of complexes 2-7, HF-SCF ${ }^{15}$ and $\mathrm{MP}_{2}{ }^{16}$ calculations have been performed on the model molecule $\left[\mathrm{HNCH}{ }_{2} \mathrm{CH}_{2} \mathrm{NHCRe}(\mathrm{NHCHCHNH})(\mathrm{CO})_{3}\right]^{+}(\mathbf{4 m})$. The calculated energy and composition of the near frontier orbitals are summarized in Table 3 and reveal that the HOMO has a high $d(R e)$ parentage $(69.5 \%)$ whereas the LUMO is dominated by $\pi^{*}$ (diimine) $(78.3 \%)$ and the percentage of $p$ (carbene $C$ ) is small (6.5\%). Figure 3 shows a simplified molecular orbital diagram of $\mathbf{4 m}$ and its component fragments $\left[(\mathrm{CO})_{3} \operatorname{Re}(\mathrm{NHCHCHNH})\right]^{+}$and $\mathrm{CNHCH}_{2} \mathrm{CH}_{2} \mathrm{NH}$. The HOMO is essentially the nonbonding $d_{x z}(R e)$ orbital, and below this are the two nonbonding $d_{y z}(R e)$ and $d_{x^{2}-y^{2}}(R e)$ orbitals $(x, y, z$ axis defined as in Figure 3); these three orbitals are very close in energy. The LUMO is comprised of $p_{z}$ (diimine) and $p_{z}$ (carbene $C$ ). Hence, if the lowest energy transition involves the frontier orbitals (see Supporting I nfor-

(19) Miessler, G. L.; Kim, S.; J acobson, R. A.; Angelici, R. J . Inorg. Chem. 1987, 26, 1690.

(20) Chen, L. C.; Chen, M. Y.; Chen, J . H.; Wen, Y. S.; Lu, K. L. J Organomet. Chem. 1992, 425, 99 .

(21) Kiel, W. A.; Lin, G. Y.; Constable, A. G.; McCormick, F. B.; Strouse, C. E.; Eisenstein, O.; Gladysz, J . A. J . Am. Chem. Soc. 1982, $104,4865$.

(22) Merrifield, J . H.; Strouse, C. E.; Gladysz, J . A. Organometallics 1982, 1, 1204.
Table 3. Calculated Energy (eV) and Composition (\%) of the Near Frontier Orbitals of Model Molecule $\left[\mathrm{HNCH} \mathrm{CH}_{2} \mathrm{NHCRe}(\mathrm{NHCHCHNH})(\mathrm{CO})_{3}\right]^{+}$ (4m) by $\mathrm{MP}_{2}$ Method

\begin{tabular}{crrrrrr}
\hline & & \multicolumn{5}{c}{ \% composition } \\
\cline { 3 - 7 } orbital & \multicolumn{1}{c}{ energy, } & \multicolumn{1}{c}{ Re } & \multicolumn{1}{c}{$\mathrm{C}$} & $\begin{array}{c}\mathrm{HNCH}_{2-} \\
\mathrm{CH}_{2} \mathrm{NH}^{-}\end{array}$ & $3 \mathrm{CO}$ & $\mathrm{CHNH}$ \\
\hline 72 & 1.265 & 14.9 & 5.4 & 4.6 & 64.2 & 11.0 \\
71 & 0.972 & 35.2 & 3.3 & 9.8 & 31.3 & 20.4 \\
70 & 0.883 & 39.4 & 12.5 & 10.4 & 22.8 & 14.9 \\
69 & 0.465 & 3.7 & 0.7 & 2.4 & 9.1 & 84.1 \\
68 & 0.177 & 56.6 & 11.0 & 5.5 & 18.6 & 8.4 \\
67 & 0.020 & 51.9 & 1.0 & 2.1 & 32.3 & 12.8 \\
66 & -0.265 & 73.6 & 6.5 & 8.2 & 6.7 & 5.0 \\
65 & -0.696 & 66.0 & 1.7 & 2.6 & 9.6 & 20.2 \\
64 & -1.192 & 69.2 & 1.9 & 4.0 & 11.3 & 13.6 \\
63 & -3.996 & 6.5 & 6.5 & 1.1 & 7.6 & 78.3 \\
62 & -12.245 & 69.5 & 0.3 & 1.7 & 19.2 & 9.2 \\
61 & -12.357 & 68.1 & 1.1 & 3.5 & 18.7 & 8.7 \\
60 & -12.584 & 73.5 & 0.2 & 0.1 & 23.5 & 2.7 \\
59 & -14.244 & 0.6 & 0.8 & 97.1 & 0.7 & 0.8 \\
58 & -15.959 & 8.6 & 47.3 & 19.6 & 13.1 & 11.4 \\
57 & -16.208 & 5.4 & 8.9 & 6.1 & 4.1 & 75.4 \\
56 & -17.848 & 1.7 & 10.2 & 59.3 & 4.5 & 24.3 \\
55 & -18.077 & 5.5 & 0.1 & 1.3 & 19.7 & 73.4 \\
54 & -18.245 & 2.2 & 2.7 & 66.2 & 7.0 & 21.9 \\
53 & -18.448 & 4.4 & 4.0 & 26.8 & 9.7 & 55.1 \\
52 & -18.993 & 4.0 & 5.6 & 15.4 & 8.3 & 66.7 \\
51 & -19.821 & 1.4 & 0.7 & 7.4 & 89.1 & 1.5 \\
50 & -19.873 & 1.7 & 0.1 & 4.0 & 90.7 & 3.5 \\
49 & -20.016 & 0.5 & 0.2 & 75.7 & 22.9 & 0.7 \\
48 & -20.030 & 1.8 & 0.3 & 16.2 & 78.6 & 3.1 \\
47 & -20.265 & 1.9 & 1.5 & 63.0 & 29.2 & 4.4 \\
46 & -20.500 & 5.3 & 0.2 & 0.2 & 86.5 & 7.8 \\
45 & -20.565 & 3.9 & 0.3 & 20.3 & 71.1 & 4.4 \\
44 & -20.710 & 5.0 & 0.5 & 7.5 & 83.5 & 3.5 \\
43 & -20.915 & 10.1 & 0.6 & 3.4 & 81.1 & 4.9
\end{tabular}

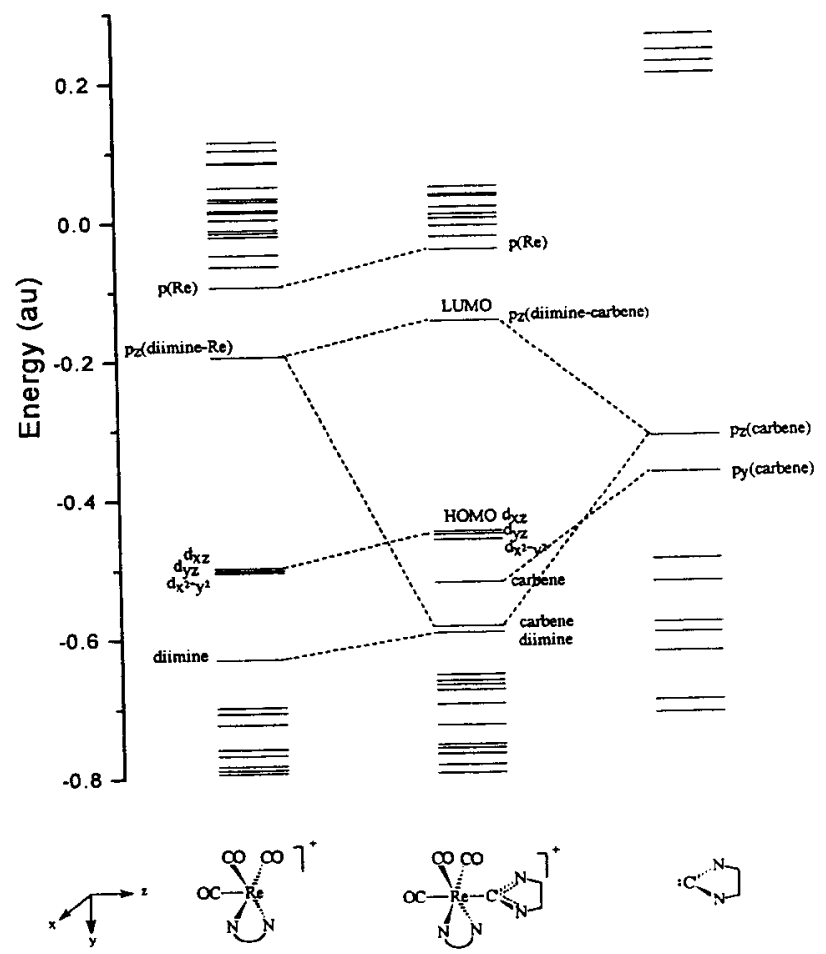

Figure 3. Simplified orbital mixing diagram for $\mathbf{4 m}$. Orbital energies are derived from $\mathrm{MP}_{2}$ calculation.

mation), then this is formulated as $\mathrm{d}(\mathrm{Re}) \rightarrow \pi^{*}$ (diimine) mixed with a small amount of $\mathrm{d}(\mathrm{Re}) \rightarrow \sigma^{*}$ (carbene $\mathrm{C}$ ).

The el ectronic absorption spectral data of complexes 1-8 are summarized in Table 4 . We suggest that 


\begin{tabular}{|c|c|c|c|c|}
\hline \multirow[b]{2}{*}{ complex } & \multicolumn{2}{|c|}{$\mathrm{CH}_{2} \mathrm{Cl}_{2}$} & \multicolumn{2}{|c|}{$\mathrm{CH}_{3} \mathrm{CN}$} \\
\hline & $\lambda, \mathrm{nm}^{\mathrm{a}}$ & $\begin{array}{c}\epsilon, 10^{3} \mathrm{dm}^{3} \\
\mathrm{~mol}^{-1} \mathrm{~cm}^{-1} \mathrm{~b}\end{array}$ & $\lambda, \mathrm{nm}^{\mathrm{a}}$ & $\begin{array}{c}\epsilon, 10^{3} \mathrm{dm}^{3} \\
\mathrm{~mol}^{-1} \mathrm{~cm}^{-1} \mathrm{~b}\end{array}$ \\
\hline 1 & $\begin{array}{l}346 \text { tail to } 560 \\
308\end{array}$ & $\begin{array}{l}3.0 \\
7.4\end{array}$ & & \\
\hline 2 & $\begin{array}{l}350 \text { tail to } 490 \\
294 \\
277 \\
255\end{array}$ & $\begin{array}{r}4.9 \\
14.6 \\
23.2 \\
36.9\end{array}$ & $\begin{array}{l}342 \\
294 \\
252\end{array}$ & $\begin{array}{r}5.8 \\
15.9 \\
47.8\end{array}$ \\
\hline 3 & $\begin{array}{l}355 \text { tail to } 500 \\
315 \\
267 \\
253\end{array}$ & $\begin{array}{r}4.5 \\
9.6 \\
20.0 \\
20.7\end{array}$ & $\begin{array}{l}345 \\
314 \\
250\end{array}$ & $\begin{array}{r}4.7 \\
9.2 \\
21.4\end{array}$ \\
\hline 4 & $\begin{array}{l}365 \text { tail to } 510 \\
318 \\
273\end{array}$ & $\begin{array}{r}3.5 \\
8.0 \\
15.4\end{array}$ & $\begin{array}{l}355 \\
316 \\
245\end{array}$ & $\begin{array}{r}4.2 \\
11.1 \\
25.5\end{array}$ \\
\hline 5 & $\begin{array}{l}380 \text { tail to } 530 \\
283 \\
255\end{array}$ & $\begin{array}{r}3.7 \\
15.5 \\
14.0\end{array}$ & $\begin{array}{l}375 \\
278 \\
253\end{array}$ & $\begin{array}{r}4.3 \\
21.1 \\
23.6\end{array}$ \\
\hline 6 & $\begin{array}{l}400 \text { tail to } 570 \\
305\end{array}$ & $\begin{array}{r}6.2 \\
21.9\end{array}$ & $\begin{array}{l}390 \\
300\end{array}$ & $\begin{array}{r}6.1 \\
21.5\end{array}$ \\
\hline 7 & $\begin{array}{l}370 \text { tail to } 510 \\
320 \\
286\end{array}$ & $\begin{array}{r}4.0 \\
6.7 \\
18.2\end{array}$ & $\begin{array}{l}360 \\
320 \\
283\end{array}$ & $\begin{array}{r}4.7 \\
8.3 \\
24.5\end{array}$ \\
\hline 8 & $\begin{array}{l}350 \\
300\end{array}$ & $\begin{array}{l}0.02 \\
2.2\end{array}$ & & \\
\hline
\end{tabular}

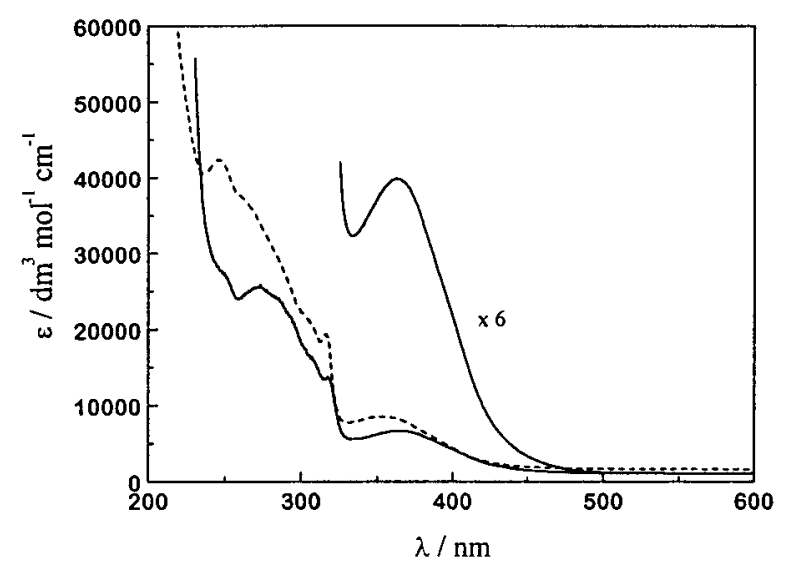

Figure 4. UV-vis absorption spectra of 4 in dichloromethane (-) and acetonitrile (- - -) at room temperature.

complex $\mathbf{1}$ has a similar electronic structure to that of $\mathrm{W}[\mathrm{C}(\mathrm{OMe}) \mathrm{Ph}](\mathrm{CO})_{5} ; 2 \mathrm{a}$ thus, the moderately intense band centered at $346 \mathrm{~nm}$ in the UV-vis absorption spectrum of $\mathbf{l}$ is assigned as the spin-allowed $d(R e) \rightarrow$ $\pi^{*}$ (carbene C) (MLCT) transition, while the very weak shoulder tailing to $560 \mathrm{~nm}$ is assigned as a spinforbidden $\mathrm{d}(\mathrm{Re}) \rightarrow \pi^{*}$ (carbene C) ( ${ }^{3} \mathrm{MLCT}$ ) transition. Replacing the $\mathrm{CO}$ ligands by diimine evidently affects the electronic structure of the carbene complexes (vide supra). The UV-vis absorption spectrum for complex 4 is shown in Figure 4.

To aid in the interpretation of the absorption spectra of these rhenium(I) carbene complexes, a single configuration interaction (CIS) calculation 23 was performed on the excited state of the optimized molecule $\mathbf{4 m}$, where all the orbitals and electrons of the ground state were included to take into account the el ectronic correlation for the excited states. The results and assignments are

(23) Foresman, J . B.; Head-Gorden, M.; Pople, J . A.; Frisch, M. J . J. Phys. Chem. 1992, 96, 135.
Table 5. Electronic Transition Energy Assignment of Optimized Model Molecule $4 \mathrm{~m}$ by CIS Calculation

\begin{tabular}{cccl}
\hline no. & $\begin{array}{c}\text { excitation } \\
\text { state }\end{array}$ & $\begin{array}{c}\lambda_{\text {calc, }} \\
\text { nm }\end{array}$ & \multicolumn{1}{c}{ assignment $^{\text {a }}$} \\
\hline 1 & triplet-A" & 433 & $62 \rightarrow 63,57 \rightarrow 63$ \\
2 & triplet-A' $^{\prime \prime}$ & 404 & $51 \rightarrow 63,61 \rightarrow 63$ \\
3 & singlet-A" & 343 & $62 \rightarrow 63$ \\
4 & triplet-A' & 328 & $60 \rightarrow 63$ \\
5 & singlet-A & 323 & $60 \rightarrow 63,61 \rightarrow 63$ \\
6 & singlet-A' & 293 & $60 \rightarrow 63,61 \rightarrow 63$ \\
7 & triplet-A' & 280 & $62 \rightarrow 64,62 \rightarrow 66,62 \rightarrow 82$ \\
8 & triplet-A" & 276 & $60 \rightarrow 64,60 \rightarrow 66,60 \rightarrow 70,60 \rightarrow 82$ \\
9 & triplet-A" & 270 & $62 \rightarrow 63,56 \rightarrow 63,57 \rightarrow 63$ \\
10 & singlet-A" & 248 & $61 \rightarrow 64,61 \rightarrow 66$ \\
11 & singlet-A' & 245 & $62 \rightarrow 64,61 \rightarrow 68,62 \rightarrow 66$ \\
12 & singlet-A" & 236 & $60 \rightarrow 64,60 \rightarrow 66,60 \rightarrow 82$ \\
13 & singlet-A" & 232 & $57 \rightarrow 63,62 \rightarrow 63,56 \rightarrow 63$ \\
14 & singlet-A" & 227 & $61 \rightarrow 66,61 \rightarrow 64,60 \rightarrow 82$ \\
\multicolumn{5}{c}{ a or the orbitals see Table 3.}
\end{tabular}

listed in Table 5. The calculated lowest energy absorption at $433 \mathrm{~nm}$ is a triplet transition of orbital $62 \rightarrow 63$ (HOMO to LUMO). This can be visualized as a spinforbidden charge-transfer transition of $d_{x z}(R e)$ to $\pi^{*}$ (diimine), where the $\pi^{*}$ (diimine) orbital has a small percentage of $p_{z}$ (carbene $C$ ), and is denoted as ${ }^{3} \mathrm{MLCT}$. From the absorption spectrum of $\mathbf{4}$, a weak shoulder around $450 \mathrm{~nm}$ correlates with this transition. The calculated HOMO to LUMO singlet transition is located at $343 \mathrm{~nm}$ and correlates with the $363 \mathrm{~nm}$ band in the absorption spectrum of $\mathbf{4}$. The intense absorption band at $273 \mathrm{~nm}$ for $\mathbf{4}$ (in $\mathrm{CH}_{2} \mathrm{Cl}_{2}$ ) may be initially assigned to the calculated transition around $293 \mathrm{~nm}$ which is an admixture of $60 \rightarrow 63$ and $61 \rightarrow 63$ transitions, namely $d_{x^{2}-y^{2}}(R e) \rightarrow \pi^{*}\left(\right.$ diimine) and $d_{y z}(R e) \rightarrow \pi^{*}($ diimine) accompanied by partial $\mathrm{d}(\mathrm{Re}) \rightarrow \mathrm{p}_{\mathrm{z}}$ (carbene $\mathrm{C}$ ) character. However, the extinction coefficient for this band ( 1.54 $\times 10^{4} \mathrm{dm}^{3} \mathrm{~mol}^{-1} \mathrm{~cm}^{-1}$ ) is much larger than that for commonly observed MLCT transitions in rhenium(I) diimine complexes. ${ }^{24}$ Furthermore, free bpy shows an intraligand $\pi \rightarrow \pi^{*}$ transition at $282 \mathrm{~nm}$ with $\epsilon_{\max }$ of $1.48 \times 10^{4} \mathrm{dm}^{3} \mathrm{~mol}^{-1} \mathrm{~cm}^{-1} .25$ Hence, it is apparent that the intense absorption of $\mathbf{4}$ at $273 \mathrm{~nm}$ is dominated by the intraligand $\pi \rightarrow \pi^{*}$ transition of the diimineligand, which is consistent with previous work on related systems. ${ }^{24}$ It should be noted that the calculated intraligand $\pi \rightarrow \pi^{*}$ transition for the "simplified 2,2'bipyridine" ligand [NHCHCHNH] in the optimized model $\mathbf{4 m}$ occurs at $232 \mathrm{~nm}$.

The singlet transition energy from HOMO to LUMO ( $\left.{ }^{1} \mathrm{MLCT}\right)$, which is assigned to the $363 \mathrm{~nm}$ band for complex 4, is affected by the electronic properties of the $4,4^{\prime}$-substituents on bpy. The LUMO is composed mainly of $\pi^{*}$ (diimine) (videsupra), so electron-donating substituents such as $\mathrm{CH}_{3} \mathrm{O}$ and t ${ }^{\mathrm{Bu}}$ will destabilize the LUMO while electron-withdrawing groups such as $\mathrm{Cl}$ and $\mathrm{CO}_{2} \mathrm{Me}$ will lower the LUMO energy. Thus, a correlation between the ${ }^{1} \mathrm{MLCT}$ transition energy and the Hammett parameters $(\sigma)$ of the substituents on bpy is anticipated and subsequently demonstrated (see Supporting Information). The ${ }^{1} \mathrm{MLCT}$ transitions of

(24) (a) Lees, A. J . Chem. Rev. 1987, 87, 711. (b) Sacksteder, L. Zipp, A. P.; Brown, E. A.; Streich, J .; Demas, J . N.; DeGraff, B. A. Inorg Chem. 1990, 29, 4335. (c) Zipp, A. P.; Sacksteder, L.; Streich, J .; Cook, A.; Demas, J. N.; DeGraff, B. A. Inorg. Chem. 1993, 32, 5629. (d) Leasure, R. M.; Sacksteder, L.; Nessel rodt, D.; Reitz, G. A.; Demas, J . N.; DeGraff, B. A. Inorg. Chem. 1991, 30, 3722. 
Table 6. Corrected Emission Maxima, Quantum Yields, and Lifetimes for Complexes 2-7 in Dichloromethane and Acetonitrile at Room Temperature

\begin{tabular}{cccccccr}
\hline & \multicolumn{3}{c}{$\mathrm{CH}_{2} \mathrm{Cl}_{2}$} & & \multicolumn{3}{c}{$\mathrm{CH}_{3} \mathrm{CN}$} \\
\cline { 2 - 3 } \cline { 6 - 8 } complex & $\lambda_{\text {max }}, \mathrm{nm}^{\mathrm{a}}$ & $\phi_{\mathrm{em}}{ }^{\mathrm{b}}$ & $\tau, \mathrm{ns}^{\mathrm{b}}$ & & $\lambda_{\max }, \mathrm{nm}^{\mathrm{a}}$ & $\phi_{\mathrm{em}}{ }^{\mathrm{b}}$ & $\tau, \mathrm{ns}^{\mathrm{b}}$ \\
\hline $\mathbf{2}$ & 558 & 0.040 & 194 & & 570 & 0.012 & 64 \\
$\mathbf{3}$ & 553 & 0.088 & 330 & & 565 & 0.038 & 150 \\
$\mathbf{4}$ & 565 & 0.068 & 230 & & 577 & 0.021 & 97 \\
$\mathbf{5}$ & 603 & 0.0075 & 45 & & 615 & 0.0020 & 20 \\
$\mathbf{6}$ & 620 & 0.015 & 97 & & 635 & 0.0039 & 40 \\
$\mathbf{7}$ & 555 & 0.30 & 2520 & & 565 & 0.11 & 932
\end{tabular}

a Error $= \pm 2 \mathrm{~nm}$. ${ }^{\mathrm{b}}$ Error $= \pm 10 \%$.

2-6 (Table 4) red-shift as the electron-withdrawing ability of the substituents increases, and this provides further support for the $d(R e) \rightarrow \pi^{*}$ (diimine) (MLCT) assignment of the absorption band.

The MLCT absorption maxima are solvent-dependent and blue-shift ca. $10 \mathrm{~nm}$ when the solvent is changed from dichloromethane to acetonitrile (Table 4). This solvatochromic effect is a characteristic feature of MLCT transitions. The shift to higher energy in solvents of greater polarity is indicative of a polar ground state and a nonpolar excited state. This is in direct contrast to the effect observed for diimine complexes such as Ru(bpy) ${ }_{3}{ }^{2+}$ and related systems, where the MLCT band maxima shift to lower energy with increasing solvent polarity typifies a nonpolar ground state and a more polar excited state. ${ }^{26}$ Similar negative solvatochromic shifts have previously been observed for the chargetransfer-to-dithiolate and charge-transfer-to-diimine absorption bands of platinum(II) dithiolate $27 \mathrm{a}$ and diimine dithiolate ${ }^{27 b}$ complexes, respectively.

Emission Spectra and Nature of the Excited States. Photoluminescence has been recorded for complexes 2-7 in fluid solution and frozen-solvent glasses. The room-temperature emission data are listed in Table 6 , while Figure 5 depicts the emission spectra of 4 (top) and 7 (bottom), respectively, in dichloromethane and acetonitrile at room temperature and in 4:1 ethanol/ methanol glass at $77 \mathrm{~K}$. In room-temperature fluid solution, the emission bands are broad and unstructured. The emitting excited states are assigned as $d_{x z}$ $(\mathrm{Re}) \rightarrow \pi^{*}$ (diimine) with partial $d_{x z}(R e) \rightarrow p_{z}($ carbene $C)$ on the basis of a linear relationship between the emission and ${ }^{1} \mathrm{MLCT}$ absorption energies (see Supporting I nformation). The relativel y long lifetimes indicate that the transitions involved are spin-forbidden.

The emission energy can be tuned by varying the diimine ligand. The 4,4'-substituents $X$ on the bpy chromophore are expected to strongly affect the MLCT excited-state energy. The carbene complexes with electron-donating substituents on bpy should, therefore, have a greater energy gap between the excited and ground states than those with electron-withdrawing substituents. Hence, a linear correlation between the emission energy and Hammett parameter for $X$ is observed (Figure 6). The shift of the emission band to lower energies for more electron-withdrawing substit-

(25) Xue, W. M.; Che, C. M. Unpublished work.

(26) Ford, W. E.; Calvin, M. Chem. Phys. Lett. 1980, 76, 105

(27) (a) Bevilacqua, J. M.; Zuleta, J . A.; Eisenberg, R. Inorg. Chem. 1993, 32, 3689. (b) Cummings, S. D.; Eisenberg, R. J. Am. Chem. Soc 1996, 118, 1949 .
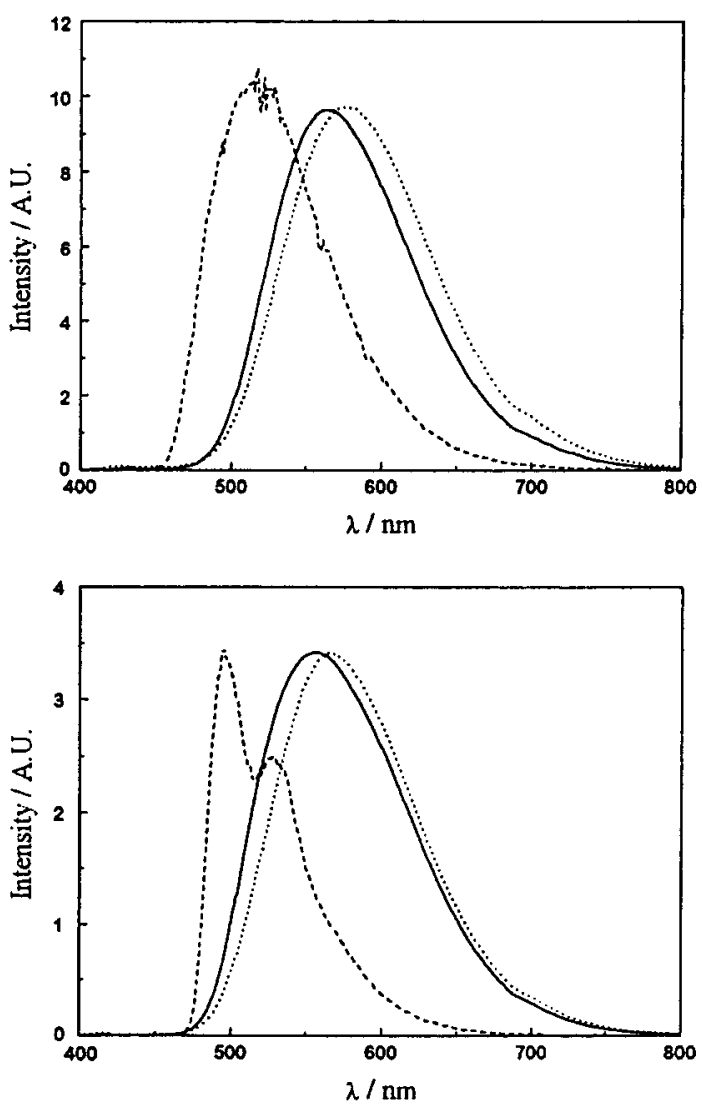

Figure 5. (top) E mission spectra of $\mathbf{4}$ in dichloromethane $\left(-, \lambda_{\mathrm{ex}}=365 \mathrm{~nm}\right)$ and acetonitrile $\left(\cdots, \lambda_{\mathrm{ex}}=355 \mathrm{~nm}\right)$ at room temperature and in 4:1 ethanol/methanol glass (- - - , $\lambda_{\text {ex }}=350 \mathrm{~nm}$ ) at $77 \mathrm{~K}$. The emission intensities are normalized. (bottom) Emission spectra of $\mathbf{7}$ in dichloromethane $\left(-, \lambda_{\mathrm{ex}}=370 \mathrm{~nm}\right)$ and acetonitrile $\left(\cdots, \lambda_{\mathrm{ex}}=370\right.$ $\mathrm{nm})$ at room temperature and in 4:1 ethanol/methanol glass $\left(---, \lambda_{\mathrm{ex}}=350 \mathrm{~nm}\right)$ at $77 \mathrm{~K}$. The emission intensities are normalized.

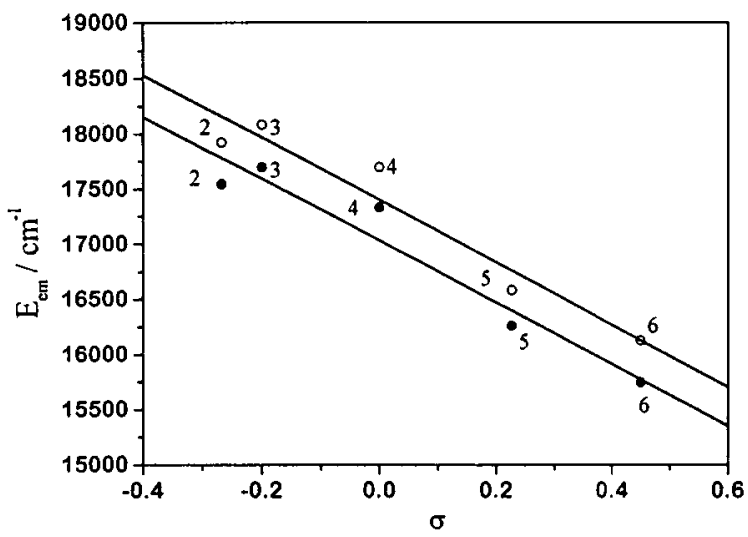

Figure 6. Room-temperature emission energies vs Hammett parameters correlation plots. Complexes are numbered as indicated in Scheme 1. Open circles indicate data in dichloromethane (correlation coefficient $R=0.97$, slope $\left.=-(2.8 \pm 0.4) \times 10^{3}\right)$, and closed circles represent data in acetonitrile $\left(\mathrm{R}=0.97\right.$, slope $\left.=-(2.8 \pm 0.4) \times 10^{3}\right)$.

uents $X$ further supports the assignment of a chargetransfer-to-diimine emitting state. Emission energies are observed to be solvent-dependent, and this is characteristic of MLCT excited states. Emission energies recorded in acetonitrile are red-shifted by ca. 400 $\mathrm{cm}^{-1}$ compared to those in dichloromethane. 

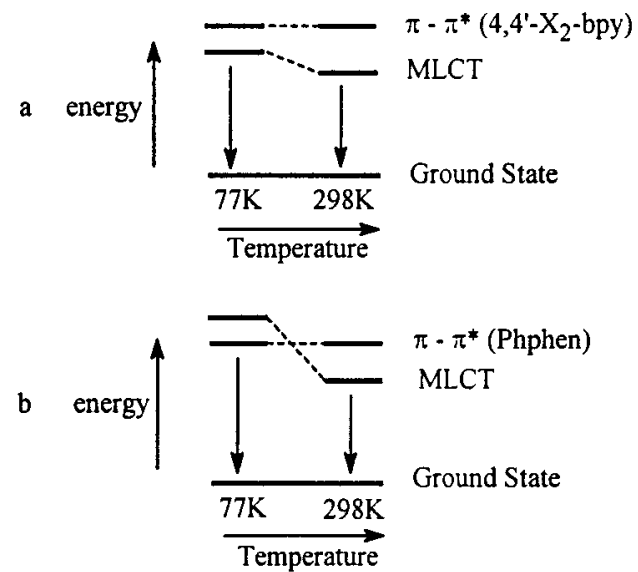

Figure 7. State diagram for the lowest triplet states of (a) $\left[\mathrm{HNCH}{ }_{2} \mathrm{CH}_{2} \mathrm{NHCRe}\left(4,4^{\prime}-\mathrm{X}_{2} \text {-bpy) }(\mathrm{CO})_{3}\right]^{+}(\mathbf{2}-6)\right.$ and (b) $\left.\left[\mathrm{HNCH}{ }_{2} \mathrm{CH}_{2} \mathrm{NHCRe} \text { Phphen)(CO) }\right]_{3}\right]^{+}$(7).

The emission spectra of complexes 2-6 measured at $77 \mathrm{~K}$ in frozen glasses are also broad and virtually structureless, but the emission maxima are shifted to higher energies than at room temperature. However, complex 7 exhibits a structured emission with peaks at 495 and $528 \mathrm{~nm}$ in 4:1 ethanol/methanol glass at $77 \mathrm{~K}$. This is clearly phosphorescence of the Phphen ligand ( $\left.{ }^{3} \mathrm{LC}\right)$, as indicated by the vibronic structure and its close resemblance to the phosphorescence of the free protonated ligand which has peak maxima at 494 and 522 $\mathrm{nm}{ }^{24 \mathrm{~b}}$ Similar emission changes with temperature and ligand had been observed by Zipp, Demas, and DeGraff for rhenium(I ) diimine complexes. ${ }^{24 b, c}$ Weadhere to their explanation using the model shown in Figure 7 . The 298 and $77 \mathrm{~K}$ emissive behavior of 2-6 belong to the model in Figure 7a, where all emissions are MLCT. The polar MLCT state energy depends strongly on the solvent organization, while the less polar IL state energy is relatively insensitive to solvent properties. At room temperature, the complex and environment can relax to the thermally equilibrated excited state in a shorter time than the emission lifetime, and this significantly lowers the MLCT state energy. However, in a rigid 77 $\mathrm{K}$ glass, solvent viscosity prevents thermal equilibration in a time comparable to the emission lifetime. On the other hand, the emissions of $\mathbf{7}$ can be represented by the model in Figure 7b. In this case, the emission is MLCT at $298 \mathrm{~K}$ but the increase in the MLCT state energy upon cooling means that the MLCT-IL state gap is smaller, and the lowest excited state is IL at $77 \mathrm{~K}$. Apart from the Re(I ) derivatives mentioned above, ${ }^{24}$ the complexity of multiple luminescences has been encountered for the $\alpha$-diimine complexes of a number of transition metals, including $\mathrm{I}(\mathrm{III}),{ }^{28} \mathrm{Cu}(\mathrm{I}),{ }^{29} \mathrm{~W}(0)$, and $\mathrm{Mo}(0){ }^{30}$ Complex 8, bearing the phosphine ligand pdpp instead of diimine, does not emit at room temperature

(28) (a) Watts, R. J ; Brown, M. J .; Griffith, B. G.; Harrington, J . S. J Am. Chem. Soc. 1975, 97, 6029. (b) Watts, R. J .; Griffith, B. G.; Harrington, J. S. J. Am. Chem. Soc. 1976, 98, 674.

(29) (a) Buckner, M. T.; Matthews, T. G.; Lytle, F. E.; McMillin, D. R. J . Am. Chem. Soc. 1979, 101, 5846. (b) Rader, R. A.; McMillin, D. R.; Buckner, M. T.; Matthews, T. G.; Casadonte, D. J .; Lengel, R. K.; Whittaker, S. B.; Darmon, L. M.; Lytle, F. E. J . Am. Chem. Soc. 1981, 103, 5906. (c) Casadonte, D. J ., J r.; McMillin, D. R. J . Am. Chem. Soc. 1987, 109, 331.

(30) (a) Manuta, D. M.; Lees, A. J . Inorg. Chem. 1983, 22, 572. (b) Manuta, D. M.; Lees, A. J . Inorg. Chem. 1986, 25, 1354.
Table 7. Electrochemical Data and Excited-State Redox Potentials of Complexes 2-7 in Acetonitrile

\begin{tabular}{cccccc}
\hline complex & $\begin{array}{c}\mathrm{E}_{1 / 2, \mathrm{ox}} \\
\left(\Delta \mathrm{E}_{\mathrm{p}}\right)^{\mathrm{a}}\end{array}$ & $\begin{array}{c}\mathrm{E}_{1 / 2, \mathrm{red}} \\
\left(\Delta \mathrm{E}_{\mathrm{p}}\right)^{\mathrm{a}}\end{array}$ & $\begin{array}{c}\mathrm{E}_{\max }, \\
\mathrm{eV}\end{array}$ & $\begin{array}{c}\mathrm{E}_{1 / 2}(2+/+*), \\
\mathrm{V}\end{array}$ & $\begin{array}{c}\mathrm{E}_{1 / 2}(+* / 0), \\
\mathrm{V}\end{array}$ \\
\hline $\mathbf{2}$ & $+1.50(90)$ & $-1.38(80)$ & 2.48 & -0.98 & +1.10 \\
$\mathbf{3}$ & $+1.56(70)$ & $-1.36(70)$ & 2.51 & -0.95 & +1.15 \\
$\mathbf{4}$ & $+1.60(90)$ & $-1.26(70)$ & 2.41 & -0.81 & +1.15 \\
$\mathbf{5}$ & $+1.62(90)$ & $-1.04(50)$ & 2.28 & -0.66 & +1.24 \\
& & $-1.62^{\mathrm{b}}$ & & & \\
$\mathbf{6}$ & $+1.66(120)$ & $-1.73^{\mathrm{b}}$ & & & \\
& & $-1.86(60)$ & 2.24 & -0.58 & +1.38 \\
$\mathbf{7}$ & $+1.59(90)$ & $-1.31(50)$ & $2.51^{\mathrm{c}}$ & -0.92 & +1.20 \\
$\mathbf{1}$ & $+1.52^{\mathrm{b}}$ & & & &
\end{tabular}

a Potentials in volts vs SCE, estimated as $1 / 2\left(E_{p a}+E_{p c}\right)$, where $E_{p a}$ and $E_{p c}$ are the potentials of peak anodic and cathodic current, respectively; $\Delta E_{p}=E_{p c}-E_{p a}{ }^{b}$ Irreversible. ${ }^{c} E_{0-0}$.

in the solution or solid state, but luminescence is observed $\left(\lambda_{\max } 460 \mathrm{~nm}\right)$ in a frozen $\mathrm{CH}_{2} \mathrm{Cl}_{2}$ solution at $77 \mathrm{~K}$ (see Supporting Information). This emission is independent of the excitation wavelength and is assigned to the pdpp I L transition.

Electrochemistry and Excited-State Redox Properties. Cyclic voltammetry is used to determine the ground-state redox potentials for complexes 2-7 (Table 7). A quasireversible oxidation wave in the +1.50 to $+1.66 \mathrm{~V}$ range (vS SCE) is recorded, which becomes irreversible when the scan speed is decreased from 500 to $100 \mathrm{mV} \mathrm{s}^{-1}$. This couple is assigned to a metalcentered oxidation, i.e., $\operatorname{Re}(\mathrm{I}) \rightarrow \operatorname{Re}(\mathrm{II}){ }^{31}$ One or more reduction waves are observed between -0.86 and -1.73 $\mathrm{V}$, the first of which is reversible while the second and third are irreversible or quasireversible. With reference to electrochemical studies on related rhenium(I) derivatives, $24 b, 31,32$ this first couple is assigned to a ligandcentered $\mathrm{L}-\mathrm{L}^{0 /-}$ reduction.

Both of the ground-state redox potentials $E_{1 / 2}\left(\mathrm{Re}^{2+/+}\right)$ and $\mathrm{E}_{1 / 2}\left(\mathrm{~L}-\mathrm{L}^{\mathrm{O} /-}\right)$ are systematically varied with different diimine ligands. The pl ots of $E_{1 / 2}\left(R^{2+/+}\right)$ and $E_{1 / 2}(L-$ $\mathrm{L}^{0 /-}$ ) of complexes 2-6 vs the Hammett parameters of the 4,4'-substituents give linear correlations (see Supporting Information). Since the substituents on bpy are not expected to affect the HOMO, which is predominantly comprised of the Re $5 d$ orbital, the variation of oxidation potential $\mathrm{E}_{1 / 2}\left(\mathrm{Re}^{2+/+}\right)$ vs $\sigma$ is less notable compared to that for $E_{1 / 2}\left(L_{-} \mathrm{L}^{0 /-}\right.$ ) (slope $0.19 \pm 0.03 \mathrm{cf}$. $0.74 \pm 0.05 \mathrm{~V}$, respectively).

A comparison of the electrochemical and emission data gives additional support for the assignment of the $\mathrm{Re} \rightarrow \pi^{*}$ (diimine) charge-transfer excited state. A linear relationship is observed between the energy of the excited state and the difference between the ground-state oxidation and reduction potentials (see Supporting Information). Similar correlations have been reported for a number of transition-metal diimine complexes. ${ }^{27 b, 32,33}$

(31) Wallace, L.; Rillema, D. P. Inorg. Chem. 1993, 32, 3836

(32) J uris, A.; Campagna, S.; Bidd, I.; Lehn, J . M.; Ziessel, R. Inorg. Chem. 1988, 27, 4007.

(33) (a) Hino, J. K.; Ciana, L. D.; Dressick, W. J .; Sullivan, B. P. Inorg. Chem. 1992, 31, 1072. (b) J uris, A.; Balzani, V.; Barigelletti, F.; Campagna, S.; Belser, P.; Von Zelewsky, A. Coord. Chem. Rev. 1988 84, 85. (c) Rillema, D. P.; Allen, G.; Meyer, T. J .; Conrad, D. Inorg. Chem. 1983, 22, 1617. (d) Caspar, J . V.; Meyer, T. J . Inorg. Chem. 1983, 22, 2444. (e) Rezvani, A. R.; Crutchley, R. J . I norg. Chem. 1994, 33,170 . 
It is al so possible to estimate the excited-state redox potentials using the following equations:

$$
\begin{gathered}
\mathrm{E}_{1 / 2}\left(2+/+^{*}\right)=\mathrm{E}_{1 / 2}(2+/+)-\mathrm{E}_{\max } \\
\mathrm{E}_{1 / 2}(+* / 0)=\mathrm{E}_{1 / 2}(+/ 0)+\mathrm{E}_{\max }
\end{gathered}
$$

where $\mathrm{E}_{1 / 2}\left(2+/+^{*}\right)$ and $\mathrm{E}_{1 / 2}(+* / 0)$ refer to the following reactions, respectively:

$$
\begin{gathered}
{\left[(\text { carbene }) \operatorname{Re}^{\prime \prime}(\mathrm{L}-\mathrm{L})^{-}(\mathrm{CO})_{3}\right]^{+*} \rightarrow} \\
{\left[(\text { carbene }) \operatorname{Re}^{\prime \prime}(\mathrm{L}-\mathrm{L})(\mathrm{CO})_{3}\right]^{2+}+\mathrm{e}^{-}} \\
{\left[(\text {carbene }) \operatorname{Re}^{\prime \prime}(\mathrm{L}-\mathrm{L})^{-}(\mathrm{CO})_{3}\right]^{+*}+\mathrm{e}^{-} \rightarrow} \\
{\left[(\text { carbene }) \operatorname{Re}^{\prime}(\mathrm{L}-\mathrm{L})^{-}(\mathrm{CO})_{3}\right]}
\end{gathered}
$$

Due to the broad structureless nature of the $77 \mathrm{~K}$ emissive bands for complexes 2-6, their $\mathrm{E}_{0-0}$ values are difficult to determine and, therefore, their respective emission maxima at $77 \mathrm{~K}$ in 4:1 ethanol-methanol glass are used as approximations for $\mathrm{E}_{0-0}$. In the case of complex 7, vibronic structure is observed in the $77 \mathrm{~K}$ emission, thus the highest energy vibrational peak is taken as $\mathrm{E}_{0-0}$. It is also important to note that any effects arising from differences in the solvent used for the el ectrochemical and spectroscopic experiments are not taken into account. Due to these factors, only the relative trends in the calculated excited-state redox potentials should be considered. The results of the calculations are presented in Table 7. The redox potentials for excited states acting as reducing agents, $\mathrm{E}_{1 / 2}\left(2+/+^{*}\right)$, varies from $-0.98 \mathrm{~V}$ for $\mathbf{2}$ to $-0.58 \mathrm{~V}$ for $\mathbf{6}$. This reflects the tendency for the ground-state oxidation potentials $E_{1 / 2,0 x}$ to increase and the $E_{0-0}$ values to decrease as the electron-accepting ability of diimine ligands increases. Variations for $\mathrm{E}_{1 / 2}(+* / 0)$ are less significant because both the ground-state reduction potential and $\mathrm{E}_{0-0}$ decrease as the 4,4'-substituted bpy becomes more electron-withdrawing. The changes in the excited-state redox potentials for the series generally correl ate with the respective $\mathrm{H}$ ammett parameters (see Supporting Information). In this investigation, the excited state of complex 2 containing 4,4'-( $\mathrm{MeO})_{2}$-bpy is the most powerful reductant $\left(\mathrm{E}_{1 / 2}\left(2+/+^{*}\right)=-0.98 \mathrm{~V}\right)$ while the excited state of 6 bearing 4,4'-( $\left.\mathrm{CO}_{2} \mathrm{Me}\right)_{2}$-bpy is the strongest oxidant $\left(\mathrm{E}_{1 / 2}(+* / 0)=+1.20 \mathrm{~V}\right)$.

\section{Conclusion}

A series of luminescent rhenium(I) complexes of the type $\left[\mathrm{HNCH} \mathrm{CH}_{2} \mathrm{NHCRe}(\mathrm{L}-\mathrm{L})(\mathrm{CO})_{3}\right]^{+}(\mathrm{L}-\mathrm{L}=$ diimine and diphosphine) have been prepared and characterized by X-ray analysis. Assignment of their lowest-lying excited states is established by investigating their emissive properties at room temperature and $77 \mathrm{~K}$ and by interpretation of molecular-orbital calculations. At room temperature, the excited state of complexes 2-7 with diimine ligands is assigned as being metal-ligand charge-transfer (MLCT) in nature and is proposed to be predominantly $d(R e) \rightarrow \pi^{*}$ (diimine) with partial $d(R e)$ $\rightarrow \sigma^{*}$ (carbene C). At $77 \mathrm{~K}$, the emitting state of complexes 2-6 remains MLCT but that of $\mathbf{7}$ bearing the Phphen ligand changes to intraligand (IL). Complex 8 with the pdpp ligand does not emit at room temperature, but at $77 \mathrm{~K}$ the pdpp IL emission is observed. Excitedand ground-state redox potentials, excited-state energies, emission lifetimes, and quantum yields can be tuned by changing the electron-donating/accepting ability of the auxiliary diimine ligand.

Acknowledgment. We thank The University of Hong Kong, the Croucher Foundation, and the Hong Kong Research Grants Council for financial support. M.C.-W.C. is grateful for a University Postdoctoral Fell owship from The University of Hong Kong. We are indebted to one of the reviewers for hel pful suggestions.

Supporting Information Available: ORTEP diagram of 4, HOMO and LUMO of $\mathbf{4 m}$, absorption energy vs Hammett parameter plots, emission energy vs ${ }^{1} \mathrm{MLCT}$ absorption energy plots, emission spectrum of $\mathbf{8}$, ground state redox potential vs Hammett parameter plot, correlation of emission energies with $\Delta \mathrm{E}_{1 / 2}$, and excited state redox potential vs Hammett parameter plots and tables of crystal data, atomic coordinates, calculated hydrogen coordinates, anisotropic displacement parameters, and bond distances and angles for 4, 6, and 8 (36 pages). Ordering information is given on any current masthead page.

OM9709042 